\title{
Combined prognostic value of CD274 (PD-L1)/PDCDI (PD-1) expression and immune cell infiltration in colorectal cancer as per mismatch repair status
}

\author{
Maarit Ahtiainen $^{1} \cdot$ Erkki-Ville Wirta $^{2,3} \cdot$ Teijo Kuopio $^{4,5} \cdot{\text { Toni Seppälä } \mathbb{D}^{6} \cdot \text { Juha Rantala }^{7} \text { Jukka-Pekka Mecklin }}^{1,8}$. \\ Jan Böhm ${ }^{4}$
}

Received: 3 August 2018 / Revised: 14 January 2019 / Accepted: 15 January 2019 / Published online: 5 February 2019

(c) United States \& Canadian Academy of Pathology 2019

\begin{abstract}
The CD274 (programmed cell death ligand-1, PD-L1)/PDCD1 (programmed cell death-1, PD-1) pathway is crucial suppressor of the cytotoxic immune response. Antibodies targeting CD274 or PDCD1 have been revealed to be effective in several malignancies. In colorectal cancer, the response to CD274/PDCD1 blockage is associated with microsatellite instability. However, the value of CD274/PDCD1 for predicting response to treatment or survival benefit is still unclear. The aims of the study were (1) to clarify differences in immune microenvironment and expression of checkpoint proteins (CD274/PDCD1) in DNA mismatch repair-proficient, mismatch repair-deficient, and hereditary Lynch syndrome-associated colorectal cancer, and (2) to assess the prognostic value of these factors and their combinations. Ninety-four mismatch repair-deficient colorectal cancers, 100 age, sex, and AJCC/UICC stage-matched mismatch repair-proficient colorectal cancers, and 48 Lynch syndrome-associated colorectal cancers were analyzed. Using whole section samples, detailed analysis of immune cell score, PDCD1, and CD274 expression was performed. Overlapping of CD274 expression in tumor and immune cells was almost complete (95\%). Immune cell score and CD274/PDCD1 positivity were significantly more frequent in mismatch repair-deficient than in mismatch repair-proficient colorectal cancers (70\% vs. $41 \%$ (high immune cell score); $81 \%$ vs. $49 \%$ (PDCD1 ${ }^{\text {high }}$ ), $23 \%$ vs. $1 \%$ (CD274 on tumor cells) and $68 \%$ vs. $30 \%$ (CD274 on immune cells), $P<$ 0.001 ), and were associated strongly with each other. Although the independent impact of immune cell score, PDCD1, and CD274 on immune cells was moderate, the immunoprofile parameter combining the above three factors appeared to be a strong independent prognostic factor for disease-specific survival and overall survival $(P=0.001)$ and had suggestive impact on disease-free survival $(P=0.011)$. Our results encourage the use of immune cell score analysis together with PDCD1 and CD274 detection to improve the prognostic evaluation of colorectal cancer patients. Particularly, the analyses from whole tissue sections are encouraged to allow reliable and cell-specific analyses of CD274 expression.
\end{abstract}

These authors contributed equally: Maarit Ahtiainen, Erkki-Ville Wirta.

Maarit Ahtiainen

maarit.ahtiainen@ksshp.fi

1 Department of Education and Research, Central Finland Central Hospital, Jyväskylä, Finland

2 Department of Surgery, Central Finland Central Hospital, Jyväskylä, Finland

3 Department of Gastroenterology and Alimentary Tract Surgery, Tampere University Hospital, Tampere, Finland

4 Department of Pathology, Central Finland Central Hospital, Jyväskylä, Finland

\section{Introduction}

Immune blockade targeting CD274 (programmed cell death ligand-1, PD-L1)/PDCD1 (programmed cell death, PD-1) is

5 Biological and Environmental Science, University of Jyväskylä, Jyväskylä, Finland

6 Department of Gastrointestinal Surgery, Abdominal Center, Helsinki University Hospital, and University of Helsinki, Helsinki, Finland

7 Cancer Research, Misvik Biology, Turku, Finland

8 Faculty of Sport and Health Sciences, University of Jyväskylä, Jyväskylä, Finland 
a promising therapeutic strategy for various malignancies $[1,2]$. The CD274/PDCD1 pathway normally serves as a negative regulator of $\mathrm{T}$ cells by preventing overstimulation of physiological immune responses to produce tolerance. Overexpression of CD274 is commonly observed in malignant cells and represents one mechanism for cancer cell immune evasion. The expression of CD274 can also be driven by constitutive oncogenic signaling pathways (e.g., enhanced expression of CD274 on glioblastomas when PTEN is silenced), although this mechanism is less frequently encountered [3]. Upregulation of CD274 on cancer cells and/or on myeloid cells together with tumor-infiltrating lymphocytes expressing PDCD1 forms a basis for immune checkpoint blockade; this blockade occurs when the interaction between these proteins is blocked by antibodies, which in turn leads to restored T-cell activity. In colorectal cancers, the efficacy of CD274/PDCD1 treatment has been shown in tumors with microsatellite instability [4-9].

Microsatellite instability arises from deficient DNA mismatch repair function and results in increased mutational burden and increased expression of various neoantigens. Microsatellite instability has been detected in approximately $15 \%$ of colorectal cancers [10]. The sporadic microsatelliteunstable phenotype usually results from epigenetic silencing of the MLH1 gene, whereas germline mutations of one of the mismatch repair genes (MLH1, MSH2, MSH6, and PMS2) is typical in hereditary Lynch syndrome [11-13]. In general, patients with microsatellite-unstable tumors have a more favorable prognosis than do patients with microsatellite-stable tumors, which has been explained by strong cytotoxic lymphocyte infiltrates resulting from high mutational burden and neoantigen load [14-17]. Neoantigen load seems to correlate with the number of tumor-infiltrating lymphocytes in microsatellite-unstable colorectal cancer $[18,19]$, as well as in hypermutated microsatellite-stable colorectal cancer [20, 21]. Several immune checkpoint-related proteins have also been shown to be overexpressed in microsatellite-unstable tumors compared with microsatellite-stable tumors, most probably as an adaptive resistance to immune response [22]; this aspect may help explain the observed response to checkpoint blockade in microsatellite-unstable colorectal cancer [6, 8, 9].

However, it is somewhat unclear as to how to identify colorectal cancer patients who will benefit from CD274/PDCD1 treatments. Patients with tumors expressing CD274 seem to have higher response rates, but the lack of CD274 does not always exclude effective treatment [23]. In addition, pre-existing antitumor immune response is regarded as essential for successful CD274/PDCD1 pathway blockade [3]. Although microsatellite-unstable colorectal cancers usually have abundant lymphocytes, microsatelliteunstable status as such seems to be an insufficient predictor of CD274/PDCD1 blockage, with the observed response rate of $40 \%$ or less [6, 8]. Multifactorial biomarker panels including tumor mutational burden, $\mathrm{CD}^{+} \mathrm{T}$-cell density, and CD274 expression are suggested to have more predictive value [24] and therefore, classification of tumors into groups based on their CD274 status and the existence of tumor-infiltrating lymphocytes has been proposed to predict treatment response [25, 26].

Regarding the overall outcome in colorectal cancer patients, the abundance of tumor-infiltrating lymphocytes has significant prognostic value regardless of tumor mismatch repair status both in local and advanced colorectal cancer [27-31]. Immunoscore ${ }^{\circledR}$ is a quantitative classification system that describes cytotoxic immune reaction, and has been launched in conjunction with a diagnostic assay to guide treatment strategies for colorectal cancer patients, and to improve the prognostic impact of tumor, node and metastases classification [32, 33]. In contrast to tumorinfiltrating lymphocytes, assessment of the CD274/PDCD1 axis is more complicated because it involves various immunohistochemical assays and evaluation methods. As a result, the prognostic value of CD274/PDCD1 is yet unclear [34-39].

With these considerations, we aimed to make an in-depth whole tumor section analysis of PDCD1 and CD274 expression, and of immune cell infiltration; we further sought to clarify the differences in three different colorectal cancer types, i.e., mismatch repair-proficient, mismatch repair-deficient, and Lynch syndrome-associated colorectal cancers. Furthermore, we assessed the prognostic value of these factors and their combinations.

\section{Materials and methods}

\section{Tumor sampling}

Our study material was obtained as part of a previously described study by Seppälä et al. [40], which included 1088 colorectal cancer patients who underwent surgery at the Central Hospital of Central Finland in Jyväskylä during the years 2000-2010. A total of 94 mismatch repair-deficient tumors, as well as equal-sized age-, sex-, and The American Joint Committee on Cancer/Union Internationale Contre le Cancer (AJCC/UICC) stage-matched mismatch repairproficient controls $(n=100)$ were included in our study. Colorectal cancer samples from Lynch syndrome patients $(n=48)$ collected by two leading Lynch syndrome research centers in Finland (Helsinki University and Jyväskylä Central Hospitals) were also included in the study. All Lynch syndrome patients were confirmed carriers of a pathogenic germline variant of $M L H 1, M S H 2$, or MSH6. These were surgical samples of tumors obtained within a period from 1983 to 2012. Due to the heterogeneity of the Lynch syndrome group and since it included only one 
cancer-related death during follow-up, Lynch syndrome tumors were not included in the survival analyses.

We reviewed hospital records to extract information regarding treatment and tumor recurrence. Causes of death were reviewed and updated in December 2015 from the Finnish Cause of Death Registry. Macroscopic examination of tumor tissue was performed by an experienced histopathologist following AJCC guidelines (5th and 6th edition). Slides were reviewed according to AJCC 8th edition to verify perineural and lymphovascular invasion rate, tumor grading, as well as tumor budding. Tumor samples were graded (grades 1-4) on the basis of the percentage of glandular formation according to the traditional World Health Organization (WHO) recommendation [41] without considering mismatch repair status. Mucinous adenocarcinoma and signet ring cell carcinoma were considered poorly differentiated (grade 3) and medullary carcinoma was considered undifferentiated (grade 4). A 6-month course of adjuvant postoperative chemotherapy with either 5-fluorouracil and oral folinic acid, oral capecitabine with or without oxaliplatin, or 5-fluorouracil, folinic acid and oxaliplatin was prescribed to medically fit patients having stage III tumors or high-risk stage II disease (T4 or T3 with perineural or lymphovascular invasion).

\section{Immunohistochemical analyses}

Mismatch repair status was determined by immunohistochemical analysis for the expression of MLH1, PMS2, MSH2, and MSH6 as described previously [40].

To select the PDCD1 and CD274 antibodies for present study, a validation was performed with clones NAT105 (Dianova GmbH, Hamburg, Germany), F07 (Sino Biological Inc., Beijing, China), and SP269 (Spring Bioscience, Pleasanton, CA, USA) for PDCD1, and clones E1L3N (Cell Signaling Technology, Danvers, MA, USA), SP142 (Spring Bioscience, Pleasanton, CA, USA), 015 (Sino Biological Inc., Beijing, China), and 28-8 (Abcam, Cambridge, UK) for CD274. For validation, a tissue microarray was constructed using formalin-fixed paraffin-embedded tissues of five tumor types known to express CD274 (advanced melanoma, nonsmall cell lung cancer, Hodgkin's lymphoma, renal cell carcinoma, and bladder cancer). Three tissue microarray cores $(0.6 \mathrm{~mm}$ in diameter) were extracted from 10 representative tumor blocks of each tumor type. Staining protocols were optimized for each antibody (data not shown) before comparison. Positive control tissues for PDCD1 and CD274 immunohistochemistry were normal tonsil and placenta, respectively. All immunohistochemical staining procedures were conducted with an automated staining platform (BONDIII, Leica Biosystems). The slides were observed with a light microscope and were further scanned with a NanoZoomerXR (Hamamatsu Photonics) at $\times 40$ magnification.
Cohorts of 100 mismatch repair-proficient, 94 mismatch repair-deficient, and 48 Lynch syndrome-associated colorectal cancer tumor samples were used for in-depth analysis of PDCD1 and CD274 expression together with immune response analysis. Formalin-fixed paraffin-embedded tissue sections of $3 \mu \mathrm{m}$ thickness were used for immunohistochemical analyses. Staining for PDCD1 and CD274 was conducted with anti-PDCD1 (SP269, 1:50; Spring Bioscience) and anti-CD274 (E1L3N, 1:100; Cell Signaling Technology) antibodies, using a BOND-III stainer (Leica Biosystems). Staining for CD3 and CD8 was conducted with anti-CD3 (LN 10, 1:50; Novocastra) and anti-CD8 (SP16, 1:100; Thermo Scientific) antibodies, using a Lab Vision Autostainer 480 (ImmunoVision Technologies Inc.). Signal visualization was done by diaminobenzidine and sections were counterstained with haematoxylin. Slides were scanned with a NanoZoomer-XR (Hamamatsu Photonics) at $\times 20$ magnification.

To specify the cell types (tumor cells, B/T lymphocytes, and macrophages) expressing PDCD1 and CD274, a multilabel immunofluorescent analysis was performed with five representative mismatch repair-proficient and mismatch repair-deficient colorectal cancer samples. For parallel immunofluorescent staining, primary antibodies against CK18 (E43 1-1, AlexaFluor 488, Abcam), CD163 (10D6, Thermo Scientific), CD3, PDCD1, and CD274 (previously mentioned) were used together with secondary antibodies (anti-mouse AlexaFluor 647 (Thermo Scientific) and anti-rabbit AlexaFluor 568 (Thermo Scientific), in conjunction with a 4'6-diamidino-2-phenylindole nuclear counterstain. Sections were imaged on the Olympus ScanR Platform at $\times 20$ magnification. Single-cell nuclear and cytoplasmic fluorescent intensities were calculated using the Olympus ScanR Analysis Software.

\section{Scoring}

CD274 expression was evaluated on tumor cells and tumorinfiltrating immune cells throughout the tumor center and the invasive front. Both the number of stained cells (percent of either tumor cells and/or immune cells stained for CD274) and the staining intensity were scored. Tumor samples were defined as CD274 positive $\left(\mathrm{CD} 274^{\mathrm{pos}}\right)$ when $>5 \%$ of the tumor cells and/or tumor-infiltrating immune cells were positive for CD274 with moderate or strong intensity. The CD274 staining intensity was scored using a scale of $0-3$ as follows: 0 for no staining, 1 for faint staining, 2 for moderate staining, and 3 for strong staining. The number of PDCD1-positive (PDCD1+) tumorinfiltrating lymphocytes (per $1 \mathrm{~mm}^{2}$ ) were calculated using QuPath [42]. The mean calculated area was $32.0 \mathrm{~mm}^{2}$ per slide. The area needed for obtaining a reliable mean value of PDCD1-positive cells was adjusted according to the 
heterogeneity of the number of PDCD1-positive cells in the current slide. The samples were divided into two groups (PDCD $1^{\text {low }}$ and PDCD $1^{\text {high }}$ ) based on calculated values for PDCD1-positive lymphocytes. To determine the cut-off value for PDCD1 positivity $\left(55 \mathrm{cells} / \mathrm{mm}^{2}\right.$ ) with optimal sensitivity and specificity, we used receiver operating characteristic curves drawn in relation to disease-specific 5 -year mortality.

CD8- and CD3-stained whole section samples were digitally scanned. Representative areas from the tumor center and the invasion front were selected and positively stained CD8 and CD3 lymphocytes were enumerated by using QuPath [42]. The width of the invasive front analyzed for immune cell score was considered to span $360 \mu \mathrm{m}$ into the tumor and $360 \mu \mathrm{m}$ into the healthy tissue from the visible tissue frontier as proposed by Hermitte [43]. The invasive front was selected manually using an annotation brush with a diameter of $720 \mu \mathrm{m}$. The mean analyzed areas from CD3- and CD8-stained sections were $11.5 \mathrm{~mm}^{2}$ and $11.3 \mathrm{~mm}^{2}$, respectively, for the tumor center, and $9.6 \mathrm{~mm}^{2}$ and $9.3 \mathrm{~mm}^{2}$, respectively, for the invasion front. Cut-off values for immune cell score were selected from receiver operating characteristic curves $\left(327\right.$ for $\mathrm{CD}^{+}$and 148 for $\mathrm{CD}^{+}$in the tumor center; 814 for $\mathrm{CD}^{+}$and 411 for $\mathrm{CD} 8^{+}$ in the invasive front). The immune cell score was formulated following the example of Galon et al. [44], as presented in our previous study [31]. Patients were divided into low immune cell score (scores 0-2) and high immune cell score (scores 3-4) groups for further analysis.

Combining patients based on data on tumor-infiltrating lymphocytes and CD274 expression into four distinctive groups has been proposed earlier [25, 26]. Accordingly, we classified the tumors into four groups according to immune cell score status and CD274 positivity. The subgroups were as follows: high immune cell score/CD274 ${ }^{\text {pos }}$ (adaptive immune resistance), low immune cell score/CD274 ${ }^{\text {neg }}$ (immunological ignorance), low immune cell score/CD274 ${ }^{\text {pos }}$ (intrinsic induction), and high immune cell score/CD274 ${ }^{\text {neg }}$ (tolerance). Furthermore, immune cell score and PDCD1 were combined and designated as low/high immune cell score and $\mathrm{PDCD} 1^{\text {low }} / \mathrm{PDCD} 1^{\text {high }}$, to define the following subgroups: high immune cell score/PDCD1 $1^{\text {high }}$, low immune cell score/PDCD $1^{\text {low }}$, low immune cell score/PDCD $1^{\text {high }}$, and high immune cell score/PDCD $1^{\text {low }}$. Additionally, four subgroups were formed according to $\mathrm{CD} 274^{\text {neg }} / \mathrm{CD} 274^{\text {pos }}$ and PDCD $1{ }^{\text {low }} / \mathrm{PDCD} 1^{\text {high }}$ designations.

Three of the above immunologic features (immune cell score, CD274 on immune cells, and PDCD1) were dichotomized so that patients were assigned a score of 0 for low/ negative and 1 for high/positive features. Consequently, patients were assigned a composite immunoprofile scoring with a range of $0-3$.

\section{Statistical analysis}

Categorical data were compared using the Pearson's chisquare test. The Kaplan-Meier method was used to calculate disease-free survival, disease-specific survival, and overall survival, and the log-rank test was used to compare differences. Survival times for disease-specific survival and overall survival were calculated from the date of surgery to the time of death or the end of follow-up. For disease-free survival, the endpoint was the date of cancer recurrence after radical surgery or the end of follow-up. Death within 30 days following surgery was considered postoperative, and a metastasis within 6 months after surgery was considered synchronous. Univariable and multivariable Cox proportional hazards regression models were used to analyze prognostic factors for disease-free, disease-specific, and overall survival. Only variables with a $P$-value of $<0.20$ in univariable analysis were included in the multivariable analysis with age, sex, and mismatch repair status. Among AJCC/UICC stage I patients there were no cancer deaths. Therefore, these patients were excluded from multivariable analysis for disease-specific survival. $P$-values of $<0.005$ were considered significant and $P$-values between 0.05 and 0.005 were defined as suggestive [45]. Statistical analysis was performed using IBM SPSS Statistics (version 23.0; SPSS Inc., Chicago, IL, USA).

\section{Results}

\section{Patient demographics}

A total of 242 colorectal cancer specimens were included in this study. The clinicopathological characteristics of the patients with mismatch repair-proficient $(n=100)$, mismatch repair-deficient $(n=94)$, and Lynch syndrome tumors are presented in Table 1. The median follow-up time was 7.0 years (interquartile range, 3-10 years). There were no statistically significant differences in age, sex, The American Society of Anesthesiologists (ASA) class, AJCC/UICC stage, and perineural/lymphovascular invasion between the mismatch repair-proficient and mismatch repair-deficient groups. Proximal location and poor tumor differentiation were seen more often in the mismatch repair-deficient group $(P<0.001)$. In mismatch repair-deficient tumors, mucinous histology was more frequent $(P=0.003)$ and tumor budding was less frequent $(P=0.001)$ than in mismatch repair-proficient tumors. In mismatch repair-deficient tumors, four signet ring cell carcinomas and four medullary carcinomas were found. Lynch syndrome tumors differed from sporadic instable colorectal cancers according to age, sex, tumor differentiation, and tumor location $(P<0.001)$. 
Table 1 Patient demographics

\begin{tabular}{|c|c|c|c|c|c|}
\hline & \multirow{2}{*}{$\begin{array}{l}\text { MMR- } \\
\text { proficient } \\
(N=100)\end{array}$} & \multirow{2}{*}{$\begin{array}{l}\text { MMR- } \\
\text { deficient } \\
(N=94)\end{array}$} & \multirow{2}{*}{$\begin{array}{l}\text { Lynch } \\
\text { syndrome } \\
(N=48)\end{array}$} & \multicolumn{2}{|l|}{$P$-value } \\
\hline & & & & $\begin{array}{l}\text { MMR-P } \\
\text { vs. } \\
\text { MMR-D }\end{array}$ & $\begin{array}{l}\text { MMR- } \\
\text { D vs. } \\
\text { Lynch }\end{array}$ \\
\hline \multicolumn{6}{|l|}{ Age } \\
\hline$\leq 75$ & 53 & $44(47)$ & $47(98)$ & 0.389 & $<0.001$ \\
\hline$>75$ & 47 & $50(53)$ & $1(2)$ & & \\
\hline \multicolumn{6}{|l|}{ Sex } \\
\hline Male & 36 & $26(28)$ & $28(58)$ & 0.213 & $<0.001$ \\
\hline Female & 64 & $68(72)$ & $20(42)$ & & \\
\hline \multicolumn{6}{|l|}{ ASA class } \\
\hline 1 & 5 & $5(5)$ & N/A & 0.911 & N/A \\
\hline 2 & 36 & $30(32)$ & & & \\
\hline 3 & 46 & $42(45)$ & & & \\
\hline 4 & 13 & $15(16)$ & & & \\
\hline \multicolumn{6}{|c|}{ AJCC/UICC stage } \\
\hline I & 14 & $17(18)$ & $15(33)$ & 0.404 & 0.107 \\
\hline II & 46 & $47(50)$ & $20(44)$ & & \\
\hline III & 36 & $24(26)$ & $11(24)$ & & \\
\hline IV & 4 & $6(6)$ & $0(0)$ & & \\
\hline \multicolumn{6}{|c|}{ Perineural invasion } \\
\hline No & 88 & $87(93)$ & $48(100)$ & 0.286 & 0.309 \\
\hline Yes & 12 & $7(7)$ & $0(0)$ & & \\
\hline \multicolumn{6}{|c|}{ Lymphovascular invasion } \\
\hline No & 51 & $52(55)$ & $27(56)$ & 0.547 & 0.988 \\
\hline Yes & 49 & $42(45)$ & $21(44)$ & & \\
\hline \multicolumn{6}{|l|}{ Tumor grade } \\
\hline 1 & 36 & $19(20)$ & $0(0)$ & $<0.001$ & 0.001 \\
\hline 2 & 48 & $32(34)$ & $12(25)$ & & \\
\hline 3 & 16 & $40(43)$ & $34(71)$ & & \\
\hline 4 & 0 & $3(3)$ & $2(4)$ & & \\
\hline \multicolumn{6}{|c|}{ Mucinous histology } \\
\hline $0-49 \%$ & 94 & $75(80)$ & $37(77)$ & 0.003 & 0.709 \\
\hline $50 \%-100 \%$ & 6 & $19(20)$ & $11(23)$ & & \\
\hline \multicolumn{6}{|l|}{ Budding } \\
\hline No & 55 & $72(77)$ & $38(79)$ & 0.001 & 0.812 \\
\hline Yes & 45 & $21(23)$ & $10(21)$ & & \\
\hline \multicolumn{6}{|l|}{ Tumor location } \\
\hline Proximal & 50 & $75(80)$ & $28(58)$ & $<0.001$ & $<0.001$ \\
\hline Distal & 34 & $16(17)$ & $6(13)$ & & \\
\hline Rectum & 16 & $3(3)$ & $14(29)$ & & \\
\hline \multicolumn{6}{|c|}{ Adjuvant chemotherapy } \\
\hline No & 61 & $65(69)$ & N/A & 0.234 & N/A \\
\hline Yes & 39 & $29(31)$ & & & \\
\hline
\end{tabular}

Values are no. (\%) unless otherwise stated

$M M R$ mismatch repair, $M M R-P$ mismatch repair-proficient, $M M R-D$ mismatch repair-deficient, $A S A$ The American Society of Anesthesiologists, AJCC The American Joint Committee on Cancer, UICC Union for International Cancer Control, N/A not available

\section{Immune contexture of mismatch repair-proficient, sporadic mismatch repair-deficient, and Lynch syndrome tumors}

Both CD274 tumor cell positivity and immune cell positivity were more frequently seen in mismatch repairdeficient tumors $(P<0.001$, Table 2$)$ as compared with mismatch repair-proficient colorectal cancers. CD274 on tumor cells was observed in $1 \%$ of mismatch repairproficient, $23 \%$ of mismatch repair-deficient, and $6 \%$ of Lynch syndrome tumors; CD274 on immune cells was detected in $30 \%$ of mismatch repair-proficient, $68 \%$ of mismatch repair-deficient, and $63 \%$ of Lynch syndrome tumors (Table 2). CD274 on tumor cells was suggestively less frequent in Lynch syndrome tumors compared with that in sporadic instable colorectal cancers $(P=0.016)$. Co-expression of CD274 on tumor and immune cells was seen in almost all cases (95\%). Two different staining patterns of CD274 expression were seen: marginal (Figs. 1a-c) and diffuse (Figs. 1b-d); the diffuse pattern was suggestively more common in sporadic mismatch repair-deficient colorectal cancers compared with mismatch repairproficient colorectal cancers $(P=0.041)$, and significantly more common compared with Lynch syndrome-associated tumors $(P=0.002)$.

Mismatch repair-proficient and mismatch repair-deficient groups differed significantly according to immune cell score/CD274 status $(P<0.001)$. The proportions of high immune cell score/CD274 $4^{\text {pos }}$, low immune cell score/ $\mathrm{CD} 274^{\text {pos }}$, high immune cell score/CD274 ${ }^{\text {neg }}$, and low immune cell score/CD274 ${ }^{\text {neg }}$ were $54 \%, 5 \%, 16 \%$, and $20 \%$, respectively, in the mismatch repair-deficient group, and were $16 \%, 7 \%, 25 \%$, and $52 \%$, respectively, in the mismatch repair-proficient group (Table 2). In Lynch syndrome tumors, the proportions did not differ significantly from those in sporadic mismatch repair-deficient tumors, at $44 \%, 8 \%, 29 \%$, and $19 \%$ for high immune cell score/ CD274 ${ }^{\text {pos }}$, low immune cell score/CD274 ${ }^{\text {pos }}$, high immune cell score/CD274 ${ }^{\text {neg }}$, and low immune cell score/CD274 ${ }^{\text {neg }}$, respectively $(P=0.286) \quad$ (Table 2$)$. Classification into immune cell score/PDCD1 and CD274/PDCD1 subgroups and their distributions are also shown in Table 2.

The immune cell score was higher in mismatch repairdeficient compared with that in mismatch repair-proficient colorectal cancer $(P<0.001)$ and no differences between sporadic mismatch repair-deficient and Lynch syndrome tumors were seen (Table 2). However, mismatch repairdeficient tumors contained more PDCD1-positive T lymphocytes compared with both mismatch repair-proficient and Lynch syndrome tumors $(P<0.001$ and 0.012 , respectively, Table 2).

To verify the cell types expressing CD274, a multilabel immunofluorescent analysis was performed for five representative samples from mismatch repair-deficient and mismatch repair-proficient groups showing strong immunohistochemical CD274 staining. Among the cell types analyzed (tumor cells, T cells, and macrophages), CD274 expression was found solely on tumor cells and macrophages. No discernible CD274 expression was seen on T cells (Fig. 2). 
Table 2 The immune contexture of mismatch repair-proficient, sporadic mismatch repair-deficient, and Lynch syndrome-associated tumors

\begin{tabular}{|c|c|c|c|c|c|c|}
\hline & \multirow{2}{*}{$\begin{array}{l}\text { MMR- } \\
\text { proficient } \\
(N=100)\end{array}$} & \multirow{2}{*}{$\begin{array}{l}\text { MMR- } \\
\text { deficient } \\
(N=94)\end{array}$} & \multirow{2}{*}{$\begin{array}{l}\text { Lynch } \\
\text { syndrome } \\
(N=48)\end{array}$} & \multirow{2}{*}{$\begin{array}{l}\text { Total } \\
(N=242)\end{array}$} & \multicolumn{2}{|l|}{$P$-value } \\
\hline & & & & & $\begin{array}{l}\text { MMR-P vs. } \\
\text { MMR-D }\end{array}$ & $\begin{array}{l}\text { MMR-D } \\
\text { vs. Lynch }\end{array}$ \\
\hline \multicolumn{7}{|l|}{ CD274 on tumor cells } \\
\hline Negative & 99 & $72(77)$ & $45(94)$ & $216(89)$ & \multirow[t]{2}{*}{$<0.001$} & \multirow[t]{2}{*}{0.016} \\
\hline Positive & 1 & $22(23)$ & $3(6)$ & $26(11)$ & & \\
\hline \multicolumn{7}{|l|}{ CD274 on immune cells } \\
\hline Negative & 70 & $30(32)$ & $18(37)$ & $118(49)$ & \multirow[t]{2}{*}{$<0.001$} & \multirow[t]{2}{*}{0.394} \\
\hline Positive & 30 & $64(68)$ & $30(63)$ & $124(51)$ & & \\
\hline \multicolumn{7}{|l|}{ PDCD1 } \\
\hline Low & 51 & $15(16)$ & $17(35)$ & $82(34)$ & \multirow[t]{2}{*}{$<0.001$} & \multirow[t]{2}{*}{0.012} \\
\hline High & 49 & $76(81)$ & $31(65)$ & $156(64)$ & & \\
\hline \multicolumn{7}{|l|}{ Immune cell score } \\
\hline 0 & 31 & $12(13)$ & $3(6)$ & 46 (19) & \multirow[t]{5}{*}{$<0.001$} & \multirow[t]{5}{*}{0.519} \\
\hline 1 & 17 & $4(4)$ & $5(10)$ & $24(10)$ & & \\
\hline 2 & 13 & $8(9)$ & $5(10)$ & $26(11)$ & & \\
\hline 3 & 17 & $13(14)$ & $7(15)$ & $37(15)$ & & \\
\hline 4 & 24 & $53(56)$ & $28(58)$ & $105(43)$ & & \\
\hline \multicolumn{7}{|l|}{$\mathrm{ICS} / \mathrm{CD} 274^{\mathrm{IC}}$} \\
\hline $\mathrm{ICS}^{\text {high }} / \mathrm{CD} 274^{\text {high }}$ & 16 & $51(54)$ & $21(44)$ & $88(36)$ & \multirow[t]{4}{*}{$<0.001$} & \multirow[t]{4}{*}{0.286} \\
\hline $\mathrm{ICS}^{\text {low }} / \mathrm{CD} 274^{\text {high }}$ & 7 & $5(5)$ & $4(8)$ & $16(7)$ & & \\
\hline ICS ${ }^{\text {high }} / \mathrm{CD} 274^{\text {low }}$ & 25 & $15(16)$ & $14(29)$ & $54(22)$ & & \\
\hline $\mathrm{ICS}^{\text {low }} / \mathrm{CD} 274^{\text {low }}$ & 52 & $19(20)$ & $9(19)$ & $80(33)$ & & \\
\hline \multicolumn{7}{|l|}{ ICS/PDCD1 } \\
\hline $\mathrm{ICS}^{\text {high }} / \mathrm{PDCD} 1^{\text {high }}$ & 28 & $62(66)$ & $28(58)$ & $118(49)$ & \multirow[t]{4}{*}{$<0.001$} & \multirow[t]{4}{*}{0.025} \\
\hline $\mathrm{ICS}{ }^{\text {low }} / \mathrm{PDCD} 1^{\text {high }}$ & 21 & $12(13)$ & $3(6)$ & $36(15)$ & & \\
\hline $\mathrm{ICS}^{\text {high}} / \mathrm{PDCD} 1^{\text {low }}$ & 13 & $3(3)$ & $7(15)$ & $23(10)$ & & \\
\hline $\mathrm{ICS} S^{\text {low }} / \mathrm{PDCD} 1^{\text {low }}$ & 38 & $10(11)$ & $1(2)$ & $57(24)$ & & \\
\hline \multicolumn{7}{|l|}{ CD274/PDCD1 } \\
\hline $\mathrm{CD} 274^{\text {high }} / \mathrm{PDCD} 1^{\text {high }}$ & 16 & $51(54)$ & $20(42)$ & $87(36)$ & \multirow[t]{4}{*}{$<0.001$} & \multirow[t]{4}{*}{0.090} \\
\hline $\mathrm{CD} 274^{\text {low }} / \mathrm{PDCD} 1^{\text {high }}$ & 33 & $25(27)$ & $11(23)$ & $69(29)$ & & \\
\hline $\mathrm{CD} 274^{\text {high }} / \mathrm{PDCD} 1^{\text {low }}$ & 7 & $5(5)$ & $5(10)$ & $17(7)$ & & \\
\hline $\mathrm{CD} 274^{\text {low }} / \mathrm{PDCD} 1^{\text {low }}$ & 44 & $10(11)$ & $12(25)$ & $65(27)$ & & \\
\hline \multicolumn{7}{|l|}{ Immunoprofile } \\
\hline 0 & 33 & $8(9)$ & $7(15)$ & $47(19)$ & $<0.001$ & 0.295 \\
\hline 1 & 35 & $11(12)$ & $10(21)$ & $56(23)$ & & \\
\hline 2 & 18 & $20(21)$ & $12(25)$ & $50(21)$ & & \\
\hline 3 & 14 & $48(51)$ & $19(40)$ & $81(33)$ & & \\
\hline
\end{tabular}

Values are no. (\%) unless otherwise stated

$M M R$ mismatch repair, $M M R-P$ mismatch repair-proficient, $M M R-D$ mismatch repair-deficient, $C D 274$ programmed cell death ligand-1, $I C$ immune cells, $P D C D 1$ programmed cell death protein 1, ICS immune cell score

Association between CD274/PDCD1 expression and clinicopathologic parameters in mismatch repairproficient and mismatch repair-deficient tumors

In addition to a strong association with mismatch repair deficiency $(P<0.001), \mathrm{CD} 274$ on immune cells, CD274 on tumor cells, and high frequency of PDCD1-positive T lymphocytes were associated significantly with a high immune cell score (all $P<0.001$, Table 3). Furthermore, CD274 on immune cells and PDCD1 ${ }^{\text {high }}$ statuses were suggestively associated with lower overall AJCC $(P=$ 0.010 and 0.007 , respectively). CD274 on tumor cells was 

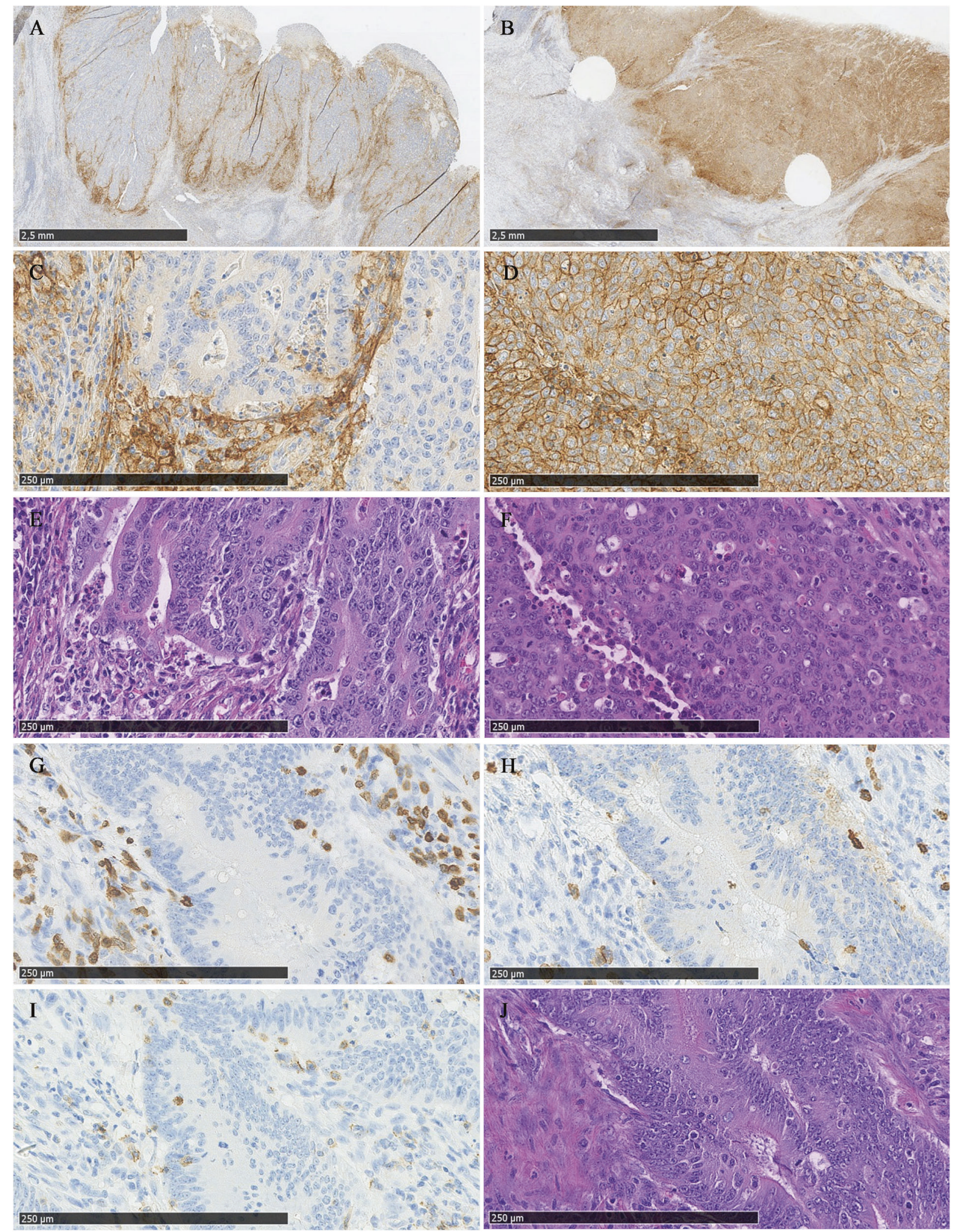

Fig. 1 Hematoxylin and eosin $(\mathrm{H} \& \mathrm{E})$ staining and immunohistochemical staining of CD274, PDCD1, CD3, and CD8 in colorectal adenocarcinomas. Two different staining patterns of CD274 expression are shown: marginal (a) and diffuse (b). $\times 20$ magnification shows $\mathrm{CD} 274^{\text {pos }}$ immune cells surrounding the tumor area (c), whereas both

tumor and immune cells are $\mathrm{CD} 274^{\text {pos }}$ in diffuse staining pattern (d) with corresponding H\&E staining (e and f, respectively). Figures g-j show representative immunostaining of CD3 (g), CD8 (h), and PDCD1 (i) and corresponding H\&E staining (j) 


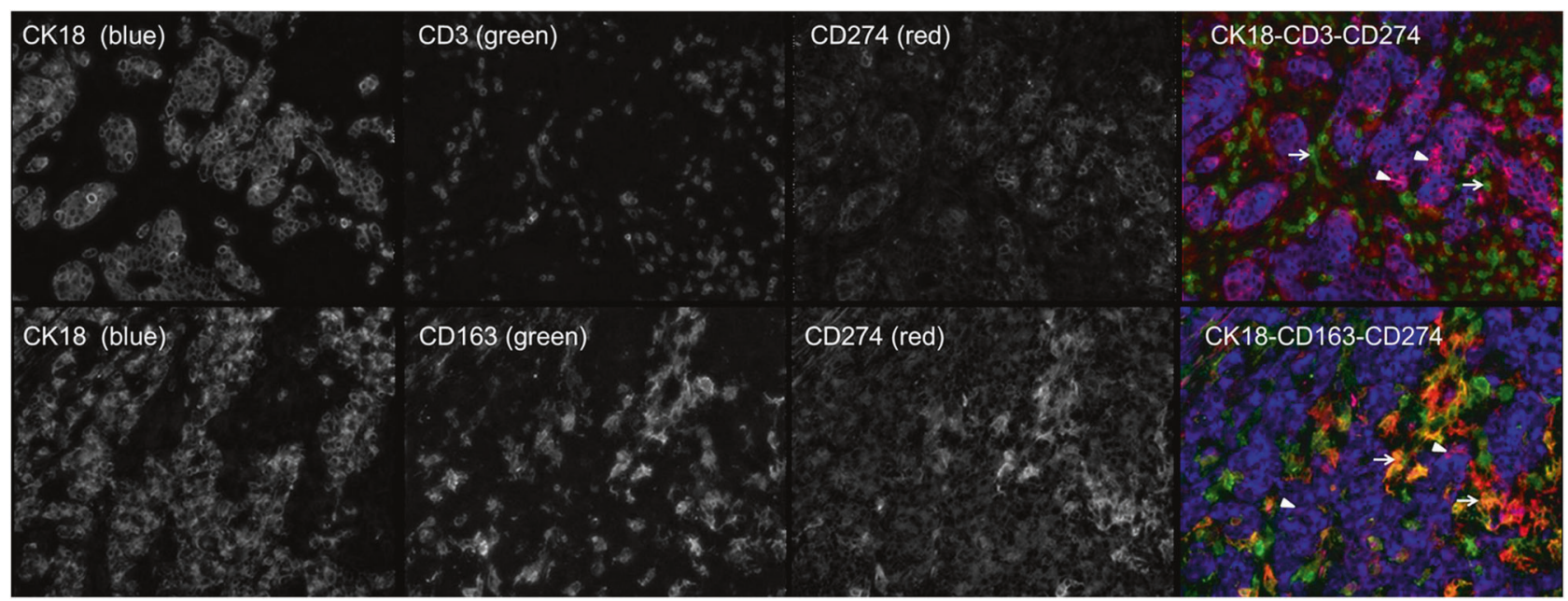

Fig. 2 Colocalization of CD274 with immune and tumor cell markers by immunofluorescence. CD274 staining in red, markers of immune cells in green (CD3 for T cells and CD163 for macrophages) and marker of tumor cells in blue (CK18). Upper panel shows the expression of CD274 in tumor cells (indicated with arrowheads). No

associated significantly and CD274 on immune cells was associated suggestively with poor differentiation $(73 \%$ grades $3-4, P<0.001$ and $41 \%$ grades $3-4, P=0.027$, respectively). CD274 on immune cells was also associated significantly and PDCD1 was associated suggestively with tumor budding $(P=0.002$ and 0.015 , respectively) and proximal tumor location $(P=0.001$ and 0.048 , respectively, Table 3). Immunoprofile, the combination of immune cell score, PDCD1 and CD274 on immune cells, did not have statistically significant associations with any of the conventional clinicopathological features in mismatch repairproficient tumors (Table 4).

\section{Prognostic impact of CD274/PDCD1 expression and immune cell infiltration}

Survival analyses according to clinicopathological parameters and immune contexture are shown in Table 5. A positive prognostic impact of immune contexture was seen both in mismatch repair-proficient and in mismatch repair-deficient colorectal cancer. In mismatch repairdeficient patients, immune cell score was significantly prognostic for 5-year disease-free, disease-specific, and overall survival (all $P<0.001$ ). For immune cell scores $0-1,2-3$, and 4 , disease-free survival was $62 \%, 95 \%$, and $95 \%$, respectively, disease-specific survival was $48 \%$, $89 \%$, and $94 \%$, respectively, and overall survival was $25 \%, 67 \%$, and $74 \%$, respectively. In mismatch repairproficient patients, immune cell score was suggestively prognostic only for disease-free survival $(P=0.010)$. CD274 on tumor cells did not affect survival of either mismatch repair-proficient or mismatch repair-deficient
CD274 expression is seen in T cells (indicated with arrows). Lower panel shows the expression of CD274 in macrophages (indicated with arrows) as well as in tumor cells (indicated with arrowheads). 20x objective

patients, whereas CD274 on immune cells had a suggestively prognostic impact on disease-specific and overall survival both in mismatch repair-proficient $(P=0.006$ and 0.011 , respectively) and mismatch repair-deficient $(P=0.048$ and 0.029 , respectively $)$ patients. $P D C D 1^{\text {high }}$ improved 5-year disease-free, disease-specific, and overall survival significantly (all $P<0.005$ ) in mismatch repairdeficient patients. In mismatch repair-proficient patients, PDCD $1^{\text {high }}$ impacted significantly on disease-free survival $(P=0.002$ and 0.004 , respectively) and suggestively on overall survival $(P=0.010)$. Furthermore, perineural invasion and tumor budding had a negative impact on disease-free, disease-specific, and overall survival $(P \leq$ 0.001 and $P \leq 0.005$, respectively) in mismatch repairproficient patients.

Our study shows that combining immune cell score, CD274 on immune cells, and PDCD1 into a single variable termed as immunoprofile more efficiently separates patients with better and worse prognosis than do any of the single immune contexture factors alone. Mismatch repair-deficient tumors with the highest immunoprofile had significantly improved 5-year disease-free, disease-specific, and overall survival (94\%, 98\%, and 77\%, respectively); mismatch repair-deficient tumors with the lowest immunoprofile had a 5 -year disease-free survival of $60 \%$, a disease-specific survival of $47 \%$, and an overall survival of $17 \%(P<0.001)$. In mismatch repair-proficient colorectal cancer patients, immunoprofile was significantly prognostic for 5-year disease-specific survival (immunoprofile $3100 \%$, immunoprofile $054 \%, P=0.004$ ) and was suggestively prognostic for disease-free and overall survival $(P=0.010$ and $P=$ 0.006, respectively, Table 5). 
Table 3 Clinicopathological and molecular characteristics according to programmed cell death ligand-1 (CD274) and programmed cell death protein 1 (PDCD1) expression

\begin{tabular}{|c|c|c|c|c|c|c|c|c|}
\hline \multicolumn{3}{|c|}{ CD274 on immune cells } & \multicolumn{3}{|c|}{ CD274 on tumor cells } & \multicolumn{3}{|l|}{ PDCD1 } \\
\hline $\begin{array}{l}\text { Negative } \\
(n=115)\end{array}$ & $\begin{array}{l}\text { Positive } \\
(n=79)\end{array}$ & $P$-value & $\begin{array}{l}\text { Negative } \\
(n=172)\end{array}$ & $\begin{array}{l}\text { Positive } \\
(n=22)\end{array}$ & $P$-value & $\begin{array}{l}\text { Low } \\
(n=65)\end{array}$ & $\begin{array}{l}\text { High } \\
(n=125)\end{array}$ & $P$-value \\
\hline
\end{tabular}

Age

$\leq 75$

$55(48) \quad 42(53)$

0.465

89 (52)

8 (36)

0.174

32 (49)

$65(52)$

0.717

$>75$

$60(52) \quad 37(47)$

83 (48) $14(64)$

$33(51)$

60 (48)

Sex

$36(31) \quad 26(33)$

0.814

58 (34)

4 (18)

0.141

21 (32)

40 (32)

0.966

Female

53 (67)

$114(66) \quad 18(82)$

$44(68)$

85 (68)

ASA

$\begin{array}{lcc}1 & 4(3) & 6(8) \\ 2 & 32(28) & 34(43) \\ 3 & 59(51) & 29(37) \\ 4 & 18(16) & 10(13)\end{array}$

0.066

$\begin{array}{ll}10(6) & 0(0) \\ 57(33) & 9(41) \\ 79(46) & 9(41) \\ 24(14) & 4(18)\end{array}$

0.582

2 (3)

8 (6)

0.603

AJCC/UICC stage

$\begin{array}{lcc}\text { I } & 20(17) & 11(14) \\ \text { II } & 44(38) & 49(62) \\ \text { III } & 44(38) & 16(20) \\ \text { IV } & 7(6) & 3(4)\end{array}$

0.010

$\begin{array}{cc}30(17) & 1(5) \\ 78(45) & 15(68) \\ 56(33) & 4(18) \\ 8(5) & 2(9)\end{array}$

0.102

21 (32)

45 (36)

$33(5) \quad 52(42)$

9 (14) 18 (14)

Perineural invasion

6 (9)

24 (19)

0.007

26 (40)

65 (52)

$26(40)$

33 (26)

7 (11)

3 (2)

$\begin{array}{lr}\text { No } & 100(87) \\ \text { Yes } & 15(13)\end{array}$

75 (95)

0.066

154 (90)

21 (95)

0.379

4 (5)

18 (10)

1 (5)

Lymphovascular invasion

\section{No}

$60(52)$

Yes

55 (48)

43 (54)

0.757

91 (53)

12 (55)

81 (47)

10 (45)

Tumor grade

$$
1
$$

$56(31)$

52 (45)

19 (24)

0.027

54 (31)

75 (44)

5 (23)

3

27 (23)

29 (37)

4

$0(0$

3 (4)

Mucinous histology

$$
\text { 0-49\% }
$$

97 (85)

70 (89)

17 (15)

50-100\%

9 (11)

Tumor budding

No

Yes

64 (57)

62 (78)

0.002

62 (36)

$18(82)$

0 (0)

3 (14)

0.481

148 (86)

19 (86)

19 (14)

$55(85)$

$116(93)$

0.074

MMR status

MMR-

proficient

64 (43)

17 (22)

77 (67)

23 (29)

$<0.001$

99 (58)

73 (42)

$21(95)$

0.885

10 (48)

$34(52)$

34 (55)

$56(45)$

deficient

$38(33) \quad 56(71)$

Immune cell score

0-1

54 (47)

2-3

37 (32)

8 (10)

$<0.001$

62 (36)

420 (17)

14 (18)

57 (72)

CD274 on immune cells

Negative
3 (14)

18 (82)

114 (66)

$1(5)$
1 (5)

13 (59)

0.981

$0(0)$

$<0.001$

35 (54)

24 (19)

$<0.001$

22 (34)

33 (26)

46 (37)

12 (18)

43 (34)

$0(0)$

3 (2)

$20(31) \quad 31(25)$

8 (12) 68 (54)

$<0.001$

$53(82)$

$58(46)$

$<0.001$ 
Table 3 (continued)

\begin{tabular}{|c|c|c|c|c|c|c|c|c|c|}
\hline & \multicolumn{3}{|c|}{ CD274 on immune cells } & \multicolumn{3}{|c|}{ CD274 on tumor cells } & \multicolumn{3}{|l|}{ PDCD1 } \\
\hline & $\begin{array}{l}\text { Negative } \\
(n=115)\end{array}$ & $\begin{array}{l}\text { Positive } \\
(n=79)\end{array}$ & $P$-value & $\begin{array}{l}\text { Negative } \\
(n=172)\end{array}$ & $\begin{array}{l}\text { Positive } \\
(n=22)\end{array}$ & $P$-value & $\begin{array}{l}\text { Low } \\
(n=65)\end{array}$ & $\begin{array}{l}\text { High } \\
(n=125)\end{array}$ & $P$-value \\
\hline Positive & & & & $58(34)$ & $21(95)$ & & $12(18)$ & $67(54)$ & \\
\hline \multicolumn{10}{|c|}{ CD274 on tumor cells } \\
\hline Negative & $114(99)$ & $58(73)$ & $<0.001$ & & & & $64(98)$ & $104(83)$ & 0.002 \\
\hline Positive & $1(1)$ & $21(27)$ & & & & & $1(2)$ & $21(17)$ & \\
\hline \multicolumn{10}{|l|}{ PDCD1 } \\
\hline Low & $53(46)$ & $12(15)$ & $<0.001$ & $64(37)$ & $1(5)$ & 0.002 & & & \\
\hline High & $58(50)$ & $67(85)$ & & $104(60)$ & $21(95)$ & & & & \\
\hline \multicolumn{10}{|c|}{ Tumor location } \\
\hline $\begin{array}{l}\text { Proximal } \\
\text { colon }\end{array}$ & $65(57)$ & $60(76)$ & 0.001 & $105(61)$ & $20(91)$ & 0.020 & $36(55)$ & $86(69)$ & 0.048 \\
\hline $\begin{array}{l}\text { Distal } \\
\text { colon }\end{array}$ & $32(28)$ & $18(23)$ & & $48(28)$ & $2(9)$ & & $18(28)$ & $31(25)$ & \\
\hline Rectum & $18(16)$ & $1(1)$ & & $19(11)$ & $0(0)$ & & $11(17)$ & $31(6)$ & \\
\hline
\end{tabular}

Values are no. (\%) unless otherwise stated

ASA The American Society of Anesthesiologists, AJCC The American Joint Committee on Cancer, UICC Union for International Cancer Control, $M M R$ mismatch repair, $P D C D 1$ programmed cell death protein 1, CD274 programmed cell death ligand-1

Multivariable analysis with the Cox proportional hazards model on separate immune parameters revealed that PDCD $1^{\text {high }}$ was an independently significant prognostic factor for disease-specific survival $(P=0.004)$ and was suggestive for disease-free and overall survival $(P=0.029$ and 0.007 , respectively, Table 6a). Additional independent prognostic factors were age for overall survival and AJCC/UICC stage for disease-specific and overall survival $(P<0.001)$. In the multivariable model where the immune parameters immune cell score, PDCD1, and CD274 on immune cells were combined, the immunoprofile was an independent prognostic factor for disease-specific and overall survival $(P=0.001)$ and was suggestive also for disease-free survival $(P=0.011$, Table $6 \mathrm{~b})$. With an immunoprofile score of 3 used as the reference, the hazard ratio (HR) for an immunoprofile score of 0 for disease-free survival was 13.01 (95\% confidence interval (CI), 2.31-73.32, $P=0.004$ ), for disease-specific survival it was 39.13 (95\% CI, 4.49-340.72, $P=0.001$ ) and for overall survival it was $3.82(95 \% \mathrm{CI}, 1.90-7.69, P<0.001)$.

\section{Discussion}

This study represents an in-depth analysis of PDCD1 and CD274 expression and immune cell infiltration together with the prognostic value of these factors in a series of mismatch repair-proficient, mismatch repair-deficient, and Lynch syndrome-associated colorectal cancers. Our results showed that immune cell infiltration and CD274/PDCD1 positivity are significantly more frequent in mismatch repair-deficient than in mismatch repair-proficient colorectal cancer, and that these factors are strongly associated with each other. In addition to tumor cells, CD274 expression was induced in inflammatory cells. CD274 positivity, either on tumor cells or on immune cells was significantly associated with high levels of cytotoxic lymphocytes in the tumor microenvironment. However, only patients with CD274 on immune cells had suggestively favorable 5-year disease-specific and overall survival despite mismatch repair status. This suggests that although considered inhibitory, PDL1 expression in immune cells is an indicator of enhanced immune activation and acts as a balancing factor of inflammatory reactions as it does during normal tolerance formation. Furthermore, the immunoprofile parameter, derived as a combination of immune cell score, PDCD1, and CD274 on immune cells, was a strong independent prognostic factor for disease-specific and overall survival, and was suggestive for disease-free survival.

Clinicopathological features analyzed in our study correspond well with those from previous studies. CD274 positivity, as well as PDCD1 density tend to associate with proximal tumor location and poor differentiation [36, 39], and these features are commonly seen in mismatch repairdeficient colorectal adenocarcinomas. In turn, tumors with high budding are more likely mismatch repair-proficient tumors [46] and show a low frequency of CD274 and PDCD1 positivity [36, 39].

Several previous studies have provided data on the expression patterns and prognostic relevance of 
Table 4 Association of immunoprofile to other clinicopathological variables in mismatch repair-proficient tumors

\begin{tabular}{|c|c|c|c|c|c|c|c|c|c|}
\hline & \multicolumn{2}{|c|}{ Immunoprofile 0} & \multicolumn{2}{|c|}{ Immunoprofile 1} & \multicolumn{2}{|c|}{ Immunoprofile 2} & \multicolumn{2}{|c|}{ Immunoprofile 3} & \multirow[t]{2}{*}{$P$-value } \\
\hline & $N$ & $\%$ & $N$ & $\%$ & $N$ & $\%$ & $N$ & $\%$ & \\
\hline \multicolumn{10}{|l|}{ Age } \\
\hline$\leq 75$ & 12 & 36 & 23 & 66 & 9 & 50 & 9 & 64 & \multirow[t]{2}{*}{0.081} \\
\hline$>75$ & 21 & 64 & 12 & 34 & 9 & 50 & 5 & 36 & \\
\hline \multicolumn{10}{|l|}{ Sex } \\
\hline Male & 9 & 27 & 13 & 37 & 6 & 33 & 8 & 57 & \multirow[t]{2}{*}{0.274} \\
\hline Female & 24 & 73 & 22 & 63 & 12 & 67 & 6 & 43 & \\
\hline \multicolumn{10}{|l|}{ ASA class } \\
\hline 1 & 1 & 3 & 1 & 3 & 1 & 6 & 2 & 14 & \multirow[t]{4}{*}{0.355} \\
\hline 2 & 7 & 21 & 14 & 40 & 9 & 50 & 6 & 43 & \\
\hline 3 & 19 & 58 & 16 & 46 & 7 & 39 & 4 & 29 & \\
\hline 4 & 6 & 18 & 4 & 11 & 1 & 6 & 2 & 14 & \\
\hline \multicolumn{10}{|l|}{ Tumor location } \\
\hline Proximal colon & 16 & 49 & 14 & 40 & 9 & 50 & 11 & 79 & \multirow[t]{3}{*}{0.283} \\
\hline Distal colon & 10 & 30 & 15 & 43 & 6 & 33 & 3 & 21 & \\
\hline Rectum & 7 & 21 & 6 & 17 & 3 & 17 & 0 & 0 & \\
\hline \multicolumn{10}{|l|}{ AJCC/UICC stage } \\
\hline I & 3 & 9 & 3 & 9 & 6 & 33 & 14 & 14 & \multirow[t]{4}{*}{0.253} \\
\hline II & 15 & 46 & 15 & 43 & 7 & 39 & 64 & 46 & \\
\hline III & 13 & 39 & 16 & 46 & 4 & 22 & 21 & 36 & \\
\hline IV & 2 & 6 & 1 & 3 & 1 & 6 & 0 & 0 & \\
\hline \multicolumn{10}{|c|}{ Perineural invasion } \\
\hline No & 28 & 85 & 28 & 80 & 18 & 100 & 14 & 100 & \multirow[t]{2}{*}{0.079} \\
\hline Yes & 5 & 15 & 7 & 20 & 0 & 0 & 0 & 0 & \\
\hline \multicolumn{10}{|c|}{ Lymphovascular invasion } \\
\hline No & 18 & 55 & 17 & 49 & 7 & 39 & 9 & 64 & \multirow{2}{*}{0.514} \\
\hline Yes & 15 & 45 & 18 & 51 & 11 & 61 & 5 & 36 & \\
\hline \multicolumn{10}{|l|}{ Tumor budding } \\
\hline No & 12 & 36 & 19 & 56 & 11 & 61 & 12 & 86 & \multirow[t]{2}{*}{0.017} \\
\hline Yes & 21 & 64 & 15 & 44 & 7 & 39 & 2 & 14 & \\
\hline \multicolumn{10}{|l|}{ Tumor grade } \\
\hline 1 & 13 & 39 & 12 & 34 & 5 & 28 & 6 & 43 & \multirow[t]{4}{*}{0.200} \\
\hline 2 & 14 & 42 & 17 & 48 & 13 & 72 & 4 & 29 & \\
\hline 3 & 6 & 18 & 6 & 17 & 0 & 0 & 4 & 29 & \\
\hline 4 & 0 & 0 & 0 & 0 & 0 & 0 & 0 & 0 & \\
\hline \multicolumn{10}{|l|}{ Mucinous tumor } \\
\hline $0-49 \%$ & 31 & 94 & 30 & 88 & 18 & 100 & 14 & 100 & \multirow[t]{2}{*}{0.261} \\
\hline $50-100 \%$ & 2 & 6 & 44 & 12 & 0 & 0 & 0 & 0 & \\
\hline
\end{tabular}

ASA The American Society of Anesthesiologists, AJCC The American Joint Committee on Cancer, UICC Union for International Cancer Control

CD274/PDCD1 in colorectal cancer [34-39], but the results have been variable and discordant. As the CD274/PDCD1 axis is involved in dynamic immune reactions, heterogeneity of immunohistochemical staining of CD274 within and between tumors is presumable, as has been shown for instance in non-small cell lung cancer, melanoma [47, 48], and recently also in colorectal cancer [35]. This can explain the variability of the results seen in studies using tissue microarrays or small biopsies for immunohistochemical analysis. To overcome this issue, we analyzed the whole section of a representative tumor block in each case. In addition, discrepant results between different studies are probably explained by differences in the CD274 antibodies used. Our decision to use clone E1L3N was based on careful validation of antibodies available. Since then, validation of commonly used antibodies has increased 
Table 5 Survival according to clinicopathological variables in mismatch repair-proficient and mismatch repair-deficient tumors

\begin{tabular}{|c|c|c|c|c|c|c|c|c|c|c|c|c|c|c|c|c|c|c|}
\hline \multirow[b]{3}{*}{ MMR status } & \multicolumn{6}{|c|}{ Disease-free survival } & \multicolumn{6}{|c|}{ Disease-specific survival } & \multicolumn{6}{|c|}{ Overall survival } \\
\hline & \multicolumn{2}{|l|}{$N$} & \multicolumn{2}{|c|}{$\begin{array}{l}\text { 5-year } \\
\text { survival }\end{array}$} & \multicolumn{2}{|l|}{$P$-value } & \multicolumn{2}{|l|}{$N$} & \multicolumn{2}{|c|}{$\begin{array}{l}\text { 5-year } \\
\text { survival }\end{array}$} & \multicolumn{2}{|l|}{$P$-value } & \multicolumn{2}{|l|}{$N$} & \multicolumn{2}{|c|}{$\begin{array}{l}5 \text {-year } \\
\text { survival }\end{array}$} & \multicolumn{2}{|l|}{$P$-value } \\
\hline & $\mathrm{P}$ & $\mathrm{D}$ & $\mathrm{P}$ & $\mathrm{D}$ & $\mathrm{P}$ & $\mathrm{D}$ & $\mathrm{P}$ & $\mathrm{D}$ & $\mathrm{P}$ & $\mathrm{D}$ & $\mathrm{P}$ & $\mathrm{D}$ & $\mathrm{P}$ & $\mathrm{D}$ & $\mathrm{P}$ & $\mathrm{D}$ & $\mathrm{P}$ & $\mathrm{D}$ \\
\hline \multicolumn{19}{|l|}{ Age } \\
\hline$\leq 75$ & 49 & 40 & $81 \%$ & $92 \%$ & 0.100 & 0.669 & 53 & 44 & $92 \%$ & $86 \%$ & $<0.001$ & 0.628 & 53 & 44 & $89 \%$ & $73 \%$ & $<0.001$ & 0.012 \\
\hline$>75$ & 39 & 43 & $57 \%$ & $88 \%$ & & & 47 & 50 & $56 \%$ & $81 \%$ & & & 47 & 50 & $43 \%$ & $54 \%$ & & \\
\hline \multicolumn{19}{|l|}{ Sex } \\
\hline Male & 31 & 23 & $84 \%$ & $100 \%$ & 0.074 & 0.107 & 36 & 26 & $82 \%$ & $92 \%$ & 0.246 & 0.306 & 36 & 26 & $75 \%$ & $54 \%$ & 0.352 & 0.148 \\
\hline Female & 57 & 60 & $64 \%$ & $87 \%$ & & & 64 & 68 & $72 \%$ & $81 \%$ & & & 64 & 68 & $63 \%$ & $66 \%$ & & \\
\hline \multicolumn{19}{|l|}{ ASA class } \\
\hline 1 & 5 & 4 & $100 \%$ & $100 \%$ & 0.050 & 0.602 & 5 & 5 & $100 \%$ & $80 \%$ & 0.002 & 0.877 & 5 & 5 & $100 \%$ & $80 \%$ & $<0.001$ & 0.399 \\
\hline 2 & 34 & 26 & $77 \%$ & $86 \%$ & & & 36 & 30 & $89 \%$ & $82 \%$ & & & 36 & 30 & $86 \%$ & $67 \%$ & & \\
\hline 3 & 40 & 38 & $69 \%$ & $89 \%$ & & & 46 & 42 & $72 \%$ & $82 \%$ & & & 46 & 42 & $63 \%$ & $62 \%$ & & \\
\hline 4 & 9 & 13 & $33 \%$ & $100 \%$ & & & 13 & 15 & $33 \%$ & $92 \%$ & & & 13 & 15 & $15 \%$ & $53 \%$ & & \\
\hline Tumor location & & & & & & & & & & & & & & & & & & \\
\hline Proximal colon & 43 & 68 & $74 \%$ & $94 \%$ & 0.332 & 0.095 & 50 & 75 & $71 \%$ & $86 \%$ & 0.438 & 0.294 & 50 & 75 & $60 \%$ & $63 \%$ & 0.357 & 0.840 \\
\hline Distal colon & 30 & 12 & $77 \%$ & $80 \%$ & & & 34 & 16 & $81 \%$ & $73 \%$ & & & 34 & 16 & $71 \%$ & $63 \%$ & & \\
\hline Rectum & 15 & 3 & $53 \%$ & $67 \%$ & & & 16 & 3 & $81 \%$ & $67 \%$ & & & 16 & 3 & $81 \%$ & $67 \%$ & & \\
\hline AJCC/UICC stag & & & & & & & & & & & & & & & & & & \\
\hline I & 13 & 16 & $100 \%$ & $93 \%$ & 0.050 & 0.130 & 14 & 17 & $100 \%$ & $100 \%$ & $<0.001$ & $<0.001$ & 14 & 17 & $93 \%$ & $53 \%$ & $<0.001$ & $<0.001$ \\
\hline II & 42 & 44 & $68 \%$ & $95 \%$ & & & 46 & 47 & $79 \%$ & $95 \%$ & & & 46 & 47 & $72 \%$ & $75 \%$ & & \\
\hline III & 33 & 23 & $64 \%$ & $82 \%$ & & & 36 & 24 & $69 \%$ & $73 \%$ & & & 36 & 24 & $58 \%$ & $63 \%$ & & \\
\hline IV & 0 & 0 & $0 \%$ & $0 \%$ & & & 4 & 6 & $0 \%$ & $0 \%$ & & & 4 & 6 & $0 \%$ & $0 \%$ & & \\
\hline Perineural invasic & & & & & & & & & & & & & & & & & & \\
\hline No & 80 & 77 & $76 \%$ & $91 \%$ & $<0.001$ & 0.518 & 88 & 87 & $79 \%$ & $85 \%$ & 0.001 & 0.472 & 88 & 87 & $72 \%$ & $62 \%$ & $<0.001$ & 0.719 \\
\hline Yes & 8 & 6 & $25 \%$ & $83 \%$ & & & 12 & 7 & $44 \%$ & $71 \%$ & & & 12 & 7 & $33 \%$ & $71 \%$ & & \\
\hline Lymphovascular & invas & ion & & & & & & & & & & & & & & & & \\
\hline No & 46 & 47 & $75 \%$ & $93 \%$ & 0.222 & 0.377 & 51 & 52 & $83 \%$ & $87 \%$ & 0.053 & 0.208 & 51 & 52 & $71 \%$ & $69 \%$ & 0.603 & 0.022 \\
\hline Yes & 42 & 26 & $66 \%$ & $86 \%$ & & & 49 & 42 & $69 \%$ & $79 \%$ & & & 49 & 42 & $63 \%$ & $55 \%$ & & \\
\hline Tumor grade & & & & & & & & & & & & & & & & & & \\
\hline 1 & 35 & 18 & $67 \%$ & $100 \%$ & 0.903 & 0.465 & 36 & 19 & $76 \%$ & $100 \%$ & 0.988 & 0.075 & 36 & 19 & $69 \%$ & $63 \%$ & 0.578 & 0.308 \\
\hline 2 & 41 & 30 & $73 \%$ & $86 \%$ & & & 48 & 32 & $73 \%$ & $86 \%$ & & & 48 & 32 & $67 \%$ & $69 \%$ & & \\
\hline 3 & 12 & 33 & $75 \%$ & $88 \%$ & & & 16 & 40 & $85 \%$ & $73 \%$ & & & 16 & 40 & $63 \%$ & $60 \%$ & & \\
\hline 4 & 0 & 2 & $100 \%$ & $100 \%$ & & & 0 & 3 & $100 \%$ & $100 \%$ & & & 0 & 3 & $100 \%$ & $33 \%$ & & \\
\hline Mucinous histolo & & & & & & & & & & & & & & & & & & \\
\hline $0-49 \%$ & 82 & 66 & $71 \%$ & $90 \%$ & 0.765 & 0.746 & 93 & 75 & $76 \%$ & $85 \%$ & 0.839 & 0.414 & 93 & 75 & $68 \%$ & $67 \%$ & 0.783 & 0.345 \\
\hline $50-100 \%$ & 5 & 17 & $80 \%$ & $92 \%$ & & & 6 & 19 & $83 \%$ & $74 \%$ & & & 6 & 19 & $67 \%$ & $47 \%$ & & \\
\hline Tumor budding & & & & & & & & & & & & & & & & & & \\
\hline No & 49 & 63 & $83 \%$ & $93 \%$ & 0.005 & 0.208 & 54 & 72 & $88 \%$ & $83 \%$ & 0.001 & 0.939 & 54 & 72 & $80 \%$ & $64 \%$ & 0.001 & 0.703 \\
\hline Yes & 38 & 19 & $57 \%$ & $80 \%$ & & & 45 & 21 & $62 \%$ & $84 \%$ & & & 45 & 21 & $53 \%$ & $57 \%$ & & \\
\hline Immune cell scor & & & & & & & & & & & & & & & & & & \\
\hline $0-1$ & 39 & 12 & $58 \%$ & $62 \%$ & 0.010 & 0.001 & 46 & 16 & $66 \%$ & $48 \%$ & 0.054 & $<0.001$ & 46 & 16 & $57 \%$ & $25 \%$ & 0.053 & $<0.001$ \\
\hline $2-3$ & 26 & 20 & $68 \%$ & $95 \%$ & & & 30 & 21 & $78 \%$ & $89 \%$ & & & 30 & 21 & $67 \%$ & $67 \%$ & & \\
\hline 4 & 23 & 49 & $96 \%$ & $95 \%$ & & & 24 & 53 & $92 \%$ & $94 \%$ & & & 24 & 53 & $88 \%$ & $74 \%$ & & \\
\hline CD274 on tumor & cells & & & & & & & & & & & & & & & & & \\
\hline No & 87 & 66 & $71 \%$ & $90 \%$ & 0.556 & 0.698 & 99 & 73 & $76 \%$ & $83 \%$ & 0.568 & 0.896 & 99 & 73 & $67 \%$ & $62 \%$ & 0.524 & 0.924 \\
\hline Yes & 1 & 17 & $100 \%$ & $93 \%$ & & & 1 & 21 & $100 \%$ & $82 \%$ & & & 1 & 21 & $100 \%$ & $67 \%$ & & \\
\hline
\end{tabular}


Table 5 (continued)

\begin{tabular}{|c|c|c|c|c|c|c|c|c|c|c|c|c|c|c|c|c|c|c|}
\hline \multirow[b]{3}{*}{ MMR status } & \multicolumn{6}{|c|}{ Disease-free survival } & \multicolumn{6}{|c|}{ Disease-specific survival } & \multicolumn{6}{|c|}{ Overall survival } \\
\hline & \multicolumn{2}{|l|}{$N$} & \multicolumn{2}{|c|}{$\begin{array}{l}\text { 5-year } \\
\text { survival }\end{array}$} & \multicolumn{2}{|c|}{$P$-value } & \multicolumn{2}{|l|}{$N$} & \multicolumn{2}{|c|}{$\begin{array}{l}\text { 5-year } \\
\text { survival }\end{array}$} & \multicolumn{2}{|c|}{$P$-value } & \multicolumn{2}{|l|}{$N$} & \multicolumn{2}{|c|}{$\begin{array}{l}\text { 5-year } \\
\text { survival }\end{array}$} & \multicolumn{2}{|c|}{$P$-value } \\
\hline & $\mathrm{P}$ & $\mathrm{D}$ & $\mathrm{P}$ & $\mathrm{D}$ & $\mathrm{P}$ & $\mathrm{D}$ & $\mathrm{P}$ & $\mathrm{D}$ & $\mathrm{P}$ & $\mathrm{D}$ & $\mathrm{P}$ & $\mathrm{D}$ & $\mathrm{P}$ & $\mathrm{D}$ & $\mathrm{P}$ & $\mathrm{D}$ & $\mathrm{P}$ & $\mathrm{D}$ \\
\hline \multicolumn{19}{|c|}{ CD274 on immune cells } \\
\hline No & 65 & 33 & $66 \%$ & $84 \%$ & 0.063 & 0.063 & 77 & 38 & $70 \%$ & $74 \%$ & 0.006 & 0.048 & 77 & 38 & $60 \%$ & $50 \%$ & 0.011 & 0.029 \\
\hline Yes & 23 & 50 & $86 \%$ & $95 \%$ & & & 23 & 56 & $96 \%$ & $90 \%$ & & & 23 & 56 & $91 \%$ & $71 \%$ & & \\
\hline \multicolumn{19}{|c|}{ PDCD1-positive cells } \\
\hline Low & 42 & 10 & $54 \%$ & $70 \%$ & 0.002 & 0.002 & 50 & 15 & $63 \%$ & $44 \%$ & 0.004 & $<0.001$ & 50 & 15 & $50 \%$ & $20 \%$ & 0.008 & $<0.001$ \\
\hline High & 46 & 70 & $87 \%$ & $95 \%$ & & & 49 & 76 & $89 \%$ & $92 \%$ & & & 49 & 76 & $86 \%$ & $72 \%$ & & \\
\hline \multicolumn{19}{|c|}{ Immunoprofile } \\
\hline 0 & 25 & 10 & $50 \%$ & $60 \%$ & 0.010 & $<0.001$ & 32 & 12 & $54 \%$ & $47 \%$ & 0.004 & $<0.001$ & 32 & 12 & $39 \%$ & $17 \%$ & 0.006 & 0.001 \\
\hline 1 & 33 & 10 & $67 \%$ & $89 \%$ & & & 35 & 14 & $79 \%$ & $66 \%$ & & & 35 & 14 & $74 \%$ & $50 \%$ & & \\
\hline 2 & 16 & 17 & $88 \%$ & $100 \%$ & & & 18 & 20 & $83 \%$ & $84 \%$ & & & 18 & 20 & $83 \%$ & $65 \%$ & & \\
\hline 3 & 14 & 46 & $100 \%$ & $94 \%$ & & & 14 & 48 & $100 \%$ & $98 \%$ & & & 14 & 48 & $93 \%$ & $77 \%$ & & \\
\hline
\end{tabular}

$M M R$ mismatch repair, $P$ mismatch repair-proficient, $D$ mismatch repair-deficient, $A S A$ The American Society of Anesthesiologists, AJCC The American Joint Committee on Cancer,UICC Union for International Cancer Control, CD274 programmed cell death ligand-1, PDCD1 programmed cell death protein 1

enormously; E1L3N is regarded as a reliable antibody and the results obtained with this antibody have been considered comparable to those obtained using the Food and Drug Administration-approved drug-specific assays [49, 50]. Further discrepancy arises from the definition of the threshold value for positive CD274 labeling. There is no consensus of what is the appropriate level of CD274 expression to separate a positive sample from a negative one. Among reported studies, CD274 positivity cut-off values vary from 1 to 50\% [51] and companion diagnostic assays include different cut-offs for defining positivity [52]. In our study, a tumor sample was defined as CD274 positive when $>5 \%$ of either tumor cells or immune cells was positive for CD274 with a moderate or strong intensity. By using the same cut-off, we obtained comparable results with previous studies on colorectal cancer using the same antibody clone (E1L3N) [34-36, 39].

In agreement with our study, Lee et al. [35] showed that CD274 expression in immune cells was associated with improved prognosis in both microsatellite-unstable and microsatellite-stable colorectal cancer, but was independent of high density of tumor-infiltrating lymphocytes. In contrast, our study showed that the prognostic impact of CD274 expression on immune cells is strongly enhanced when combined with a high immune cell score and PDCD1 positivity, which further indicates that an active immune microenvironment is counterbalanced by immune inhibition. In turn, CD274 on tumor cells did not show any prognostic impact on survival outcome in accordance with Lee's study [35]; however, this was in contrast to other studies, which showed an association of CD274 on tumor cells with worse survival [34, 36, 39]. However, all our CD274 tumor-positive patients, except for one, were simultaneously expressing CD274 on immune cells. The observed intense overlapping may have resulted from extensive analyses of whole tissue sections. The common intratumoral heterogeneity of CD274 expression in tumor and immune cells in colorectal cancer may explain the discrepancy between results from different studies.

According to the cancer immunoediting theory [53], immune evasion includes three phases, as follows: elimination, equilibrium, and escape. The expression of CD274 on tumor cells is regarded as a prominent mechanism devised to escape antitumor response. Thus, logically, CD274 tumor positivity could then mark worse prognosis. Potentially, the lack of impact on survival in our study may be due to the limited number of tumor-positive CD274 samples, although the number of CD274-positive tumor samples was also small in other studies [34, 35, 39]. This suggests that the PD1/PDL1 checkpoint mechanism is not the major escape mechanism for tumor cells in colorectal cancer, which could also explain the limited impact of anti-PDL1/PD1 treatment [6, 8]. There is evidence suggesting that local overproduction of prostaglandins such as PG2, promotes the presence of immunosuppressive and regulatory immune cells, allowing host immune evasion of tumor cells [54]. Reduction of prostaglandin production by inhibiting cyclooxygenase enzymes (COX-1 and COX-2) with aspirin therapy may reverse these effects and enhance T-cell-mediated immune response against tumor cells, although tumors with a high level of CD274 expression seem to develop aspirin resistance. Prostaglandin inhibition 
Table 6a Multivariable analysis using Cox proportional hazards model with various immune parameters

\begin{tabular}{|c|c|c|c|c|c|c|c|}
\hline & \multirow{2}{*}{$\begin{array}{l}\text { Univariable } \\
\text { analysis } \\
P\end{array}$} & \multicolumn{2}{|c|}{ Disease-free survival $(n=161)$} & \multicolumn{2}{|c|}{ Disease-specific survival $(n=147)$} & \multicolumn{2}{|c|}{ Overall survival $(n=184)$} \\
\hline & & $\mathrm{HR}(95 \% \mathrm{CI})$ & $P$ & $\mathrm{HR}(95 \% \mathrm{CI})$ & $P$ & $\mathrm{HR}(95 \% \mathrm{CI})$ & $P$ \\
\hline Age & DFS: 0.041 & & & & & & \\
\hline$<75$ & DSS: 0.002 & 1 & 0.118 & 1 & 0.015 & 1 & $<0.001$ \\
\hline$>75$ & OS: $<0.001$ & $1.94(0.84-4.47)$ & & $3.25(1.25-8.45)$ & & $3.49(2.06-5.90)$ & \\
\hline Sex & DFS: 0.052 & & & & & & \\
\hline Male & DSS: 0.198 & $0.37(0.13-1.03)$ & 0.056 & $0.48(0.18-1.28)$ & 0.143 & $1.72(1.03-2.88)$ & 0.038 \\
\hline Female & OS: 0.850 & 1 & & 1 & & 1 & \\
\hline \multicolumn{8}{|l|}{ ASA class } \\
\hline 1 & DFS: 0.864 & Excluded & 1 & 0.872 & 1 & 0.031 & \\
\hline 2 & DSS: 0.160 & & $0.66(0.08-5.76)$ & & $2.73(0.35-20,99)$ & & \\
\hline 3 & OS: $<0.001$ & & $0.91(0.11-7.73)$ & & $4.16(0.55-31.38)$ & & \\
\hline 4 & & & $0.66(0.07-6.44)$ & & $7.03(0.90-54.87)$ & & \\
\hline \multicolumn{8}{|l|}{ Tumor location } \\
\hline Proximal & DFS: 0.021 & 1 & 0.607 & Excluded & & & \\
\hline Distal & DSS: 0.778 & $1.39(0.48-4.01)$ & & & & & \\
\hline Rectum & OS: 0.357 & $1.70(0.59-4.87)$ & & & & & \\
\hline \multicolumn{8}{|l|}{ AJCC/UICC stage: } \\
\hline I & DFS: 0.028 & 1 & 0.228 & Excluded & $<0.001$ & 1 & $<0.001$ \\
\hline II & DSS: $<0.001$ & $4.28(0.50-36.38)$ & & 1 & & $0.66(0.34-1.29)$ & \\
\hline III & OS: $<0.001$ & $6.31(0.70-56.47)$ & & $1.41(0.62-3.19)$ & & $0.77(0.38-1.56)$ & \\
\hline IV & & No cases & & $31.91(10.23-99.57)$ & & $4.70(1.76-12.50)$ & \\
\hline Perineural invasion & DFS: 0.002 & & & & & & \\
\hline No & DSS: 0.007 & 1 & 0.050 & 1 & 0.084 & 1 & 0.058 \\
\hline Yes & OS: 0.004 & $2.88(1.00-8.29)$ & & $2.59(0.88-7.60)$ & & $2.00(0.98-4.08)$ & \\
\hline Lymphovascular invasion & DFS: 0.117 & & 0.327 & & 0.075 & & 0.927 \\
\hline No & DSS: 0.017 & 1 & & 1 & & 1 & \\
\hline Yes & OS: 0.057 & $1.38(0.59-4.87)$ & & $2.21(0.92-5.31)$ & & $0.98(0.60-1.58)$ & \\
\hline Tumor budding & DFS: 0.001 & & & & & & \\
\hline No & DSS: 0.005 & 1 & 0.588 & 1 & 0.762 & 1 & 0.155 \\
\hline Yes & OS: 0.007 & $1.30(0.50-3.36)$ & & $0.86(0.34-2.22)$ & & $1.43(0.87-2.35)$ & \\
\hline MMR status & DFS: 0.005 & & & & & & \\
\hline MMR-proficient & DSS: 0.127 & $1.03(0.31-3.43)$ & 0.959 & $0.70(0.26-1.91)$ & 0.485 & $0.49(0.28-0.86)$ & 0.013 \\
\hline MMR-deficient & OS: 0.638 & 1 & & 1 & & 1 & \\
\hline \multicolumn{8}{|l|}{ Immune cell score } \\
\hline $0-1$ & DFS: $<0.001$ & $5.76(1.30-25.44)$ & & $3.74(1.13-12.38)$ & & $1.93(1.00-3.73)$ & \\
\hline $2-3$ & DSS: $<0.001$ & $3.59(0.78-16.51)$ & & $2.91(0.83-10.18)$ & & $1.66(0.90-3.05)$ & \\
\hline 4 & OS: $<0.001$ & 1 & 0.062 & 1 & 0.097 & 1 & 0.132 \\
\hline CD274 on immune cells & DFS: 0.002 & & & & & & \\
\hline Low & DSS: 0.001 & $1.20(0.35-4.04)$ & 0.77 & $2.14(0.64-7.20)$ & 0.218 & $1.18(0.63-2.23)$ & 0.602 \\
\hline High & OS: 0.004 & 1 & 3 & 1 & & 1 & \\
\hline PDCD1 & DFS: $<0.001$ & & & & & & \\
\hline Low & DSS: $<0.001$ & $2.83(1.11-7.24)$ & 0.029 & $3.65(1.50-8.87)$ & 0.004 & $2.05(1.21-3.47)$ & 0.007 \\
\hline High & OS: 0.001 & 1 & & 1 & & 1 & \\
\hline
\end{tabular}

Univariate analyses were performed with the following reference categories: 75 years or under, female sex, ASA class 1, proximal tumor location, AJCC/UICC stage I (or in DSS stage II), no perineural invasion, no lymphovascular invasion, no tumor budding, mismatch repair deficiency, immune cell score 4, CD274 on immune cells and high PDCD1. Only variables with a $P$-value of $<0.20$ in univariable analysis were included in the multivariable analysis together with age and sex. Mismatch repair status was included also in overall survival analysis because of special interest in its impact. There were 161 cases available for the analysis of disease-free survival, 147 for disease-specific survival, and 184 for overall survival. Twenty-three patients were excluded from disease-free survival analysis due to either existing metastasis or non-radical surgery, and 3 patients were censored before the earliest event in a stratum. Patients with AJCC/UICC stage I disease $(n=31)$ were discarded from diseasespecific survival analysis because there was no disease-specific mortality and nine patients were censored before the earliest event in a stratum. Data pertaining to ASA class were missing for two, tumor budding was missing for 2, PDCD1 were missing for three, and immune cell score were missing for four patients

$H R$ hazard ratio, $C I$ confidence interval, ASA The American Society of Anesthesiologists, AJCC The American Joint Committee on Cancer, UICC, Union for International Cancer Control, PDCD1 programmed cell death protein 1, MMR mismatch repair, DFS disease-free survival; DSS diseasespecific survival, $O S$ overall survival 
Table 6b Multivariable analysis using the Cox proportional hazards model with the parameter immunoprofile (a parameter combining immune cell score, programmed cell death protein 1 (PDCD1), and programmed cell death ligand-1 (CD274) on immune cells)

\begin{tabular}{|c|c|c|c|c|c|c|c|}
\hline & \multirow{2}{*}{$\begin{array}{l}\text { Univariable analysis } \\
P\end{array}$} & \multicolumn{2}{|c|}{ Disease-free survival $(n=161)$} & \multicolumn{2}{|c|}{ Disease-specific survival $(n=147)$} & \multicolumn{2}{|c|}{ Overall survival $(n=184)$} \\
\hline & & $\mathrm{HR}(95 \% \mathrm{CI})$ & $P$ & $\mathrm{HR}(95 \% \mathrm{CI})$ & $P$ & HR $(95 \%$ CI $)$ & $P$ \\
\hline Age & DFS: 0.041 & & & & & & \\
\hline$<75$ & DSS: 0.002 & 1 & 0.176 & 1 & 0.016 & 1 & $<0.001$ \\
\hline$>75$ & OS: $<0.001$ & $1.79(0.77-4.18)$ & & $3.37(1.26-9.01)$ & & $3.29(1.95-5.55)$ & \\
\hline Sex & DFS: 0.052 & & & & & & \\
\hline Male & DSS: 0.198 & $0.39(0.14-1.08)$ & 0.071 & $0.58(0.22-1.53)$ & 0.271 & $1.83(1.10-3.06)$ & 0.021 \\
\hline Female & OS: 0.850 & 1 & & 1 & & 1 & \\
\hline \multicolumn{8}{|l|}{ ASA class } \\
\hline 1 & DFS: 0.864 & Excluded & & 1 & 0.960 & 1 & 0.021 \\
\hline 2 & DSS: 0.160 & & & $0.98(0.12-8.36)$ & & $4.45(0.59-33.38)$ & \\
\hline 3 & OS: $<0.001$ & & & $1.24(0.15-10.32)$ & & $3.47(0.46-26.20)$ & \\
\hline 4 & & & & $1.02(0.11-9.64)$ & & $8.66(1.11-67.33)$ & \\
\hline \multicolumn{8}{|l|}{ Tumor location } \\
\hline Proximal & DFS: 0.021 & 1 & 0.511 & Excluded & & & \\
\hline Distal & DSS: 0.778 & $1.40(0.52-3.81)$ & & & & & \\
\hline Rectum & OS: 0.357 & $1.84(0.65-5.21)$ & & & & & \\
\hline \multicolumn{8}{|l|}{ AJCC/UICC stage } \\
\hline I & DFS: 0.028 & 1 & 0.135 & Excluded & $<0.001$ & 1 & $<0.001$ \\
\hline II & DSS: $<0.001$ & $6.21(0.76-50.68)$ & & 1 & & $0.71(0.37-1.36)$ & \\
\hline III & OS: $<0.001$ & $8.73(1.02-74.77)$ & & $1.43(0.63-3.22)$ & & $0.87(0.44-1.73)$ & \\
\hline IV & & No cases & & $30.08(9.31-97.17)$ & & $6.41(2.41-17.00)$ & \\
\hline Perineural invasion & DFS: 0.002 & & & & & & \\
\hline No & DSS: 0.007 & 1 & 0.048 & 1 & 0.079 & 1 & 0.097 \\
\hline Yes & OS: 0.004 & $2.95(1.01-8.61)$ & & $2.68(0.89-8.02)$ & & $1.85(0.89-3.82)$ & \\
\hline Lymphovascular invasion & DFS: 0.117 & & & & & & \\
\hline No & DSS: 0.017 & 1 & 0.415 & 1 & 0.080 & 1 & 0.847 \\
\hline Yes & OS: 0.057 & $1.42(0.61-3.31)$ & & $2.18(0.91-5.20)$ & & $0.95(0.59-1.55)$ & \\
\hline Tumor budding & DFS: 0.001 & & & & & & \\
\hline No & DSS: 0.005 & 1 & 0.509 & 1 & 0.795 & 1 & 0.338 \\
\hline Yes & OS: 0.007 & $1.36(0.54-3.43$ & & $0.89(0.35-2.23)$ & & $1.27(0.78-2.09)$ & \\
\hline MMR status & DFS: 0.005 & & & & & & \\
\hline MMR-proficient & DSS: 0.127 & $1.08(0.35-3.36)$ & 0.890 & $0.79(0.31-2.00)$ & 0.622 & $0.53(0.31-0.92)$ & 0.024 \\
\hline MMR-deficient & OS: 0.638 & 1 & & 1 & & 1 & \\
\hline \multicolumn{8}{|l|}{ Immunoprofile } \\
\hline 0 & DFS: $<0.001$ & $13.01(2.31-73.32)$ & 0.011 & $39.13(4.49-340.72)$ & 0.001 & $3.82(1.90-7.69)$ & 0.001 \\
\hline 1 & DSS: $<0.001$ & $7.71(1.44-41.37)$ & & $22.89(2.73-191.79)$ & & $2.18(1.11-4.28)$ & \\
\hline 2 & OS: 0.001 & $1.96(0.26-14.92)$ & & $6.77(0.74-62.00)$ & & $1.00(0.49-2.03)$ & \\
\hline 3 & & 1 & & 1 & & 1 & \\
\hline
\end{tabular}

Univariate analyses were performed with the following reference categories: 75 years or under, female sex, ASA class 1, proximal tumor location, AJCC/UICC stage I (or in DSS stage II), no perineural invasion, no lymphovascular invasion, no tumor budding, mismatch repair deficiency and immunoprofile class 3 . Only variables with a $P$-value of $<0.20$ in univariable analysis were included in the multivariable analysis together with age and sex. Mismatch repair status was included also in overall survival analysis because of special interest in its impact. There were 161 cases available for analysis of disease-free survival, 147 for disease-specific survival, and 184 for overall survival. Twenty-three patients were excluded from disease-free survival analysis due to either existing metastasis or non-radical surgery. Patients with AJCC/UICC stage I disease $(n=31)$ were discarded from disease-specific survival analysis because there was no disease-specific mortality and nine patients were censored before the earliest event in a stratum. Data pertaining to ASA class were missing for two, tumor budding were missing for two, and immunoprofile were missing for seven patients

$H R$ hazard ratio, CI confidence interval, ASA The American Society of Anesthesiologists, AJCC The American Joint Committee on Cancer, UICC Union for International Cancer Control, MMR mismatch repair, DFS disease-free survival, DSS disease-specific survival, $O S$ overall survival

combined with immune checkpoint blockade therapy is therefore suggested as a possible means to improve clinical patient outcome [55].

Remarkably, CD274 on tumor cells was less common in Lynch syndrome patients compared with sporadic mismatch repair-deficient patients, as reported previously [39, 56]. Lynch syndrome colorectal cancer patients have exceptionally good prognosis even though they have an increased risk for metachronous tumors in various tissues [57, 58]. We did not see any difference in immune cell infiltration 
between sporadic mismatch repair-deficient and Lynch syndrome patients, except for a lower density of PDCD1positive lymphocytes in Lynch syndrome tumors. Whether the rarity of CD274 on tumor cells also impacts on the enhanced survival of Lynch syndrome colorectal cancer patients, remains to be studied.

Recent evidence has shown an association between sensitivity to immune checkpoint blockage and mismatch repair deficiency in colorectal cancer $[6,8]$. However, not all microsatellite-unstable colorectal cancers respond. It is currently unknown whether there is a difference in response to CD274/PDCD1 immunotherapy between CD274 expression on tumor versus immune cells in colorectal cancer. Unfortunately, our study does not enable us to correlate the staining patterns with the response to checkpoint blockade. However, several studies on murine models have shown that the expression of CD274 in host myeloid cells is highly essential for the response to checkpoint blockade, as recently reviewed by Tang and Zheng [59]. Interestingly, Zhang et al. showed that blocking both tumorand non-tumor-derived CD274 achieves synergy and maximizes antitumor T-cell response in mouse models [60]. Whether this applies to human remains to be clarified, as the number of treated colorectal cancer patients increases. In our study, one mismatch repair-proficient colorectal cancer case was highly divergent from the rest of the mismatch repair-proficient group and showed strong CD274 expression both in tumor and immune cells. It has been suggested that microsatellite-stable patients with increased CD274 expression and high immune cell score could also benefit from immune checkpoint blockage [61]. Recently, the first case of a microsatellite-stable colorectal cancer patient harboring amplification of both the CD274 and PD-L2 genes was reported; this patient showed a dramatic response to a checkpoint inhibitor [62]. Whether the exceptional CD274 expression in tumor cells of mismatch repairproficient patients seen in our study reflects amplification of the above-mentioned genes, is not known. However, these observations strongly support the importance of CD274 expression in both cell types. Large cohort of patients treated with checkpoint inhibitors need to be evaluated to confirm the predictive value of CD274/PDCD1 expression on treatment response.

Overall, our study provided data indicating that although the analyzed immune microenvironment factors in colorectal cancer were more common in mismatch repairdeficient patients, the same features in mismatch repairproficient patients were also associated with better survival. In addition to the well-known prognostic value of tumorinfiltrating $\mathrm{T}$ cells in colorectal cancer [27-31, 33], the variable immunoprofile, derived by combining immune cell score, PDCD1, and CD274, was a strong independent prognostic factor for survival. Although traditional tumor classification and risk factors like AJCC/UICC stage, tumor invasion, and patient comorbidities are recognized for their prognostic value, our results support the use of the immune cell score analysis together with PDCD1 and CD274 detection as an important prognostic variable in colorectal cancer. We also encourage the analysis of whole tissue sections for a reliable and cell-specific analysis of CD274 expression.

Acknowledgements We thank Ms. Mari Saarinen-Valta and Mr. Reino Pitkänen for technical assistance. This study received funding from the Finnish Cancer Foundation, Jane and Aatos Erkko Foundation, the State Research Funding (Kuopio University Hospital Research Center), the Finnish Medical Foundation, and the Mary and Georg C. Ehrnrooth Foundation.

\section{Compliance with ethical standards}

Conflict of interest The authors declare that they have no conflict of interest.

Ethical aspects The study was approved by the ethical committee of the central Finland Central Hospital and the National Supervisory Authority for Welfare and Health (Valvira).

Publisher's note: Springer Nature remains neutral with regard to jurisdictional claims in published maps and institutional affiliations.

\section{References}

1. Hamanishi J, Mandai M, Matsumura N, Abiko K, Baba T, Konishi I, et al. PD-1/PD-L1 blockade in cancer treatment: perspectives and issues. Int J Clin Oncol. 2016;21:462-73.

2. Homet Moreno B, Ribas A. Anti-programmed cell death protein-1/ligand-1 therapy in different cancers. Br $\mathrm{J}$ Cancer. 2015;112:1421-7.

3. Pardoll DM. The blockade of immune checkpoints in cancer immunotherapy. Nat Rev Cancer. 2012;12:252-64.

4. Brahmer JR, Drake CG, Wollner I, Powderly JD, Picus J, Sharfman $\mathrm{WH}$, et al. Phase I study of single-agent anti-programmed death-1 (MDX-1106) in refractory solid tumors: safety, clinical activity, pharmacodynamics, and immunologic correlates. J Clin Oncol. 2010;28:3167-75.

5. Brahmer JR, Tykodi SS, Chow LQM, Hwu W-J, Topalian SL, Hwu P, et al. Safety and activity of anti-PD-L1 antibody in patients with advanced cancer. N Engl J Med. 2012;366:2455-65.

6. Le DT, Uram JN, Wang H, Bartlett BR, Kemberling H, Eyring AD, et al. PD-1 Blockade in Tumors with Mismatch-Repair Deficiency. N Engl J Med. 2015;372:2509-20.

7. Topalian SL, Hodi FS, Brahmer JR, Gettinger SN, Smith DC, McDermott DF, et al. Safety, activity, and immune correlates of anti-PD-1 antibody in cancer. N Engl J Med. 2012;366:2443-54.

8. Overman MJ, McDermott R, Leach JL, Lonardi S, Lenz H-J, Morse MA, et al. Nivolumab in patients with metastatic DNA mismatch repair-deficient or microsatellite instability-high colorectal cancer (CheckMate 142): an open-label, multicentre, phase 2 study. Lancet Oncol. 2017;18:1182-91.

9. Le DT, Durham JN, Smith KN, Wang H, Bartlett BR, Aulakh LK, et al. Mismatch repair deficiency predicts response of solid tumors to PD-1 blockade. Science. 2017;357:409-13. 
10. Imai $\mathrm{K}$, Yamamoto $\mathrm{H}$. Carcinogenesis and microsatellite instability: the interrelationship between genetics and epigenetics. Carcinogenesis. 2008;29:673-80.

11. Herman JG, Umar A, Polyak K, Graff JR, Ahuja N, Issa JP, et al. Incidence and functional consequences of hMLH1 promoter hypermethylation in colorectal carcinoma. Proc Natl Acad Sci USA. 1998;95:6870-5.

12. Peltomaki P. Deficient DNA mismatch repair: a common etiologic factor for colon cancer. Hum Mol Genet. 2001;10:735-40.

13. Lynch H, Lynch P, Lanspa S, Snyder C, Lynch J, Boland C. Review of the Lynch syndrome: history, molecular genetics, screening, differential diagnosis, and medicolegal ramifications. Clin Genet. 2009;76:1-18.

14. Fridman WH, Pagès F, Sautès-Fridman $C$, Galon J. The immune contexture in human tumours: impact on clinical outcome. Nat Rev Cancer. 2012;12:298-306.

15. Nosho K, Baba Y, Tanaka N, Shima K, Hayashi M, Meyerhardt JA, et al. Tumour-infiltrating T-cell subsets, molecular changes in colorectal cancer, and prognosis: cohort study and literature review. J Pathol. 2010;222:350-66.

16. Ogino S, Nosho K, Irahara N, Meyerhardt JA, Baba Y, Shima K, et al. Lymphocytic reaction to colorectal cancer is associated with longer survival, independent of lymph node count, microsatellite instability, and $\mathrm{CpG}$ island methylator phenotype. Clin Cancer Res. 2009;15:6412-20.

17. Smyrk TC, Watson P, Kaul K, Lynch HT. Tumor-infiltrating lymphocytes are a marker for microsatellite instability in colorectal carcinoma. Cancer. 2001;91:2417-22.

18. Tougeron D, Fauquembergue E, Rouquette A, Le Pessot F, Sesboüé R, Laurent M, et al. Tumor-infiltrating lymphocytes in colorectal cancers with microsatellite instability are correlated with the number and spectrum of frameshift mutations. Mod Pathol. 2009;22:1186-95.

19. Maby P, Tougeron D, Hamieh M, Mlecnik B, Kora H, Bindea G, et al. Correlation between Density of CD8+ T-cell Infiltrate in Microsatellite Unstable Colorectal Cancers and Frameshift Mutations: A Rationale for Personalized Immunotherapy. Cancer Res. 2015;75:3446-55.

20. Angelova M, Charoentong P, Hackl H, Fischer ML, Snajder R, Krogsdam AM, et al. Characterization of the immunophenotypes and antigenomes of colorectal cancers reveals distinct tumor escape mechanisms and novel targets for immunotherapy. Genome Biol. 2015;16:64.

21. Giannakis M, Mu XJ, Shukla SA, Qian ZR, Cohen O, Nishihara $\mathrm{R}$, et al. Genomic Correlates of Immune-Cell Infiltrates in Colorectal Carcinoma. Cell Rep 2016. https://doi.org/10.1016/j. celrep.2016.03.075.

22. Llosa NJ, Cruise M, Tam A, Wicks EC, Hechenbleikner EM, Taube JM, et al. The vigorous immune microenvironment of microsatellite instable colon cancer is balanced by multiple counter-inhibitory checkpoints. Cancer Discov. 2015;5:43-51.

23. Sunshine J, Taube JM. PD-1/PD-L1 inhibitors. Curr Opin Pharmacol. 2015;23:32-38.

24. Topalian SL, Taube JM, Anders RA, Pardoll DM. Mechanismdriven biomarkers to guide immune checkpoint blockade in cancer therapy. Nat Rev Cancer. 2016;16:275-87.

25. Taube JM, Anders RA, Young GD, Xu H, Sharma R, McMiller $\mathrm{TL}$, et al. Colocalization of inflammatory response with B7-h1 expression in human melanocytic lesions supports an adaptive resistance mechanism of immune escape. Sci Transl Med. 2012;4:127ra37.

26. Teng MWL, Ngiow SF, Ribas A, Smyth MJ. Classifying Cancers Based on T-cell Infiltration and PD-L1. Cancer Res. 2015;75:2139-45.

27. Pagès F, Kirilovsky A, Mlecnik B, Asslaber M, Tosolini M, Bindea G, et al. In Situ Cytotoxic and Memory T Cells Predict
Outcome in Patients With Early-Stage Colorectal Cancer. J Clin Oncol. 2009;27:5944-51.

28. Mlecnik B, Tosolini M, Kirilovsky A, Berger A, Bindea G, Meatchi T, et al. Histopathologic-based prognostic factors of colorectal cancers are associated with the state of the local immune reaction. J Clin Oncol. 2011;29:610-8.

29. Anitei M-G, Zeitoun G, Mlecnik B, Marliot F, Haicheur N, Todosi A-M, et al. Prognostic and predictive values of the immunoscore in patients with rectal cancer. Clin Cancer Res. 2014;20:1891-9.

30. Kwak Y, Koh J, Kim DW, Kang SB, Kim WH, Lee HS. Immunoscore encompassing CD3 + and CD8 $+\mathrm{T}$ cell densities in distant metastasis is a robust prognostic marker for advanced colorectal cancer. Oncotarget. 2016;7:81778-90.

31. Wirta E-V, Seppälä T, Friman M, Väyrynen J, Ahtiainen M, Kautiainen $\mathrm{H}$, et al. Immunoscore in mismatch repair-proficient and -deficient colon cancer. J Pathol Clin Res. 2017;3:203-13.

32. Galon J, Mlecnik B, Bindea G, Angell HK, Berger A, Lagorce C, et al. Towards the introduction of the 'Immunoscore' in the classification of malignant tumours. J Pathol. 2014;232: 199-209.

33. Pagès F, Mlecnik B, Marliot F, Bindea G, Ou F-S, Bifulco C, et al. International validation of the consensus Immunoscore for the classification of colon cancer: a prognostic and accuracy study. Lancet. 2018;391:2128-39.

34. Kim JH, Park HE, Cho N-Y, Lee HS, Kang GH. Characterisation of PD-L1-positive subsets of microsatellite-unstable colorectal cancers. Br J Cancer. 2016;115:490-6.

35. Lee KS, Kwak Y, Ahn S, Shin E, Oh H-K, Kim D-W, et al. Prognostic implication of CD274 (PD-L1) protein expression in tumor-infiltrating immune cells for microsatellite unstable and stable colorectal cancer. Cancer Immunol Immunother. 2017;66:927-39.

36. Lee LH, Cavalcanti MS, Segal NH, Hechtman JF, Weiser MR, Smith JJ, et al. Patterns and prognostic relevance of PD-1 and PD-L1 expression in colorectal carcinoma. Mod Pathol. 2016;29:1-10.

37. Li Y, Liang L, Dai W, Cai G, Xu Y, Li X, et al. Prognostic impact of programed cell death-1 (PD-1) and PD-ligand 1 (PD-L1) expression in cancer cells and tumor infiltrating lymphocytes in colorectal cancer. Mol Cancer. 2016;15:55.

38. Masugi Y, Nishihara R, Yang J, Mima K, da Silva A, Shi Y, et al. Tumour CD274 (PD-L1) expression and $\mathrm{T}$ cells in colorectal cancer. Gut. 2017;66:1463-73.

39. Rosenbaum MW, Bledsoe JR, Morales-Oyarvide V, Huynh TG, Mino-Kenudson M. PD-L1 expression in colorectal cancer is associated with microsatellite instability, BRAF mutation, medullary morphology and cytotoxic tumor-infiltrating lymphocytes. Mod Pathol. 2016;29:1-9.

40. Seppälä TT, Böhm JP, Friman M, Lahtinen L, Väyrynen VMJ, Liipo TKE, et al. Combination of microsatellite instability and BRAF mutation status for subtyping colorectal cancer. $\mathrm{Br} \mathrm{J}$ Cancer. 2015;112:1966-75.

41. Hamilton SR, Aaltonen LA In: Hamilton SR, Aaltonen LA, eds. WHO Classification of Tumors. Pathology and Genetics of Tumours of Digestive System. Lyon, France: IARC Press, 2000:204. http://www.joplink.net/prev/200803/ref/08-004.html.

42. Bankhead P, Loughrey MB, Fernández JA, Dombrowski Y, McArt DG, Dunne PD, et al. QuPath: Open source software for digital pathology image analysis. Sci Rep. 2017;7:16878.

43. Hermitte F. Biomarkers immune monitoring technology primer: Immunoscore ${ }^{\circledR}$ Colon. J Immunother Cancer. 2016;4:57.

44. Galon J, Costes A, Sanchez-Cabo F, Kirilovsky A, Mlecnik B, Lagorce-Pagès C, et al. Type, Density, and Location of Immune Cells Within Human Colorectal Tumors Predict Clinical Outcome. Science (80-). 2006;313:1960-4. 
45. Benjamin DJ, Berger JO, Johannesson M, Nosek BA, Wagenmakers E-J, Berk R, et al. Redefine statistical significance. Nat Hum Behav. 2018;2:6-10.

46. Graham RP, Vierkant RA, Tillmans LS, Wang AH, Laird PW, Weisenberger DJ, et al. Tumor Budding in Colorectal Carcinoma: Confirmation of Prognostic Significance and Histologic Cutoff in a Population-based Cohort. Am J Surg Pathol. 2015;39: 1340-6.

47. Ilie M, Long-Mira E, Bence C, Butori C, Lassalle S, Bouhlel L, et al. Comparative study of the PD-L1 status between surgically resected specimens and matched biopsies of NSCLC patients reveal major discordances: a potential issue for anti-PD-L1 therapeutic strategies. Ann Oncol. 2016;27:147-53.

48. Madore J, Vilain RE, Menzies AM, Kakavand H, Wilmott JS, Hyman J, et al. PD-L1 expression in melanoma shows marked heterogeneity within and between patients: implications for anti-PD1/PD-L1 clinical trials. Pigment Cell Melanoma Res. 2015;28: 245-53.

49. Rimm DL, Han G, Taube JM, Yi ES, Bridge JA, Flieder DB, et al. A Prospective, Multi-institutional, Pathologist-Based Assessment of 4 Immunohistochemistry Assays for PD-L1 Expression in Non-Small Cell Lung Cancer. JAMA Oncol. 2017;3:1051.

50. Gaule P, Smithy JW, Toki M, Rehman J, Patell-Socha F, Cougot D, et al. A Quantitative Comparison of Antibodies to Programmed Cell Death 1 Ligand 1. JAMA Oncol. 2017;3:256.

51. Ilie M, Hofman V, Dietel M, Soria J-C, Hofman P. Assessment of the PD-L1 status by immunohistochemistry: challenges and perspectives for therapeutic strategies in lung cancer patients. Virchows Arch. 2016;468:511-25.

52. Sholl LM, Aisner DL, Allen TC, Beasley MB, Borczuk AC, Cagle PT, et al. Programmed Death Ligand-1 Immunohistochemistry- A New Challenge for Pathologists: A Perspective From Members of the Pulmonary Pathology Society. Arch Pathol Lab Med. 2016;140:341-4.
53. Dunn GP, Bruce AT, Ikeda H, Old LJ, Schreiber RD. Cancer immunoediting: from immunosurveillance to tumor escape. Nat Immunol. 2002;3:991-8.

54. Wang D, DuBois RN. The Role of Prostaglandin E(2) in TumorAssociated Immunosuppression. Trends Mol Med. 2016;22:1-3.

55. Hamada T, Cao Y, Qian ZR, Masugi Y, Nowak JA, Yang J, et al. Aspirin Use and Colorectal Cancer Survival According to Tumor CD274 (Programmed Cell Death 1 Ligand 1) Expression Status. J Clin Oncol. 2017;35:1836-44.

56. Shiraliyeva N, Friedrichs J, Buettner R, Friedrichs N. PD-L1 expression in HNPCC-associated colorectal cancer. Pathol - Res Pract. 2017;213:1552-5.

57. Drescher KM, Sharma P, Lynch HT. Current hypotheses on how microsatellite instability leads to enhanced survival of Lynch Syndrome patients. Clin Dev Immunol. 2010;2010:170432.

58. Møller P, Seppälä T, Bernstein I, Holinski-Feder E, Sala P, Evans DG, et al. Incidence of and survival after subsequent cancers in carriers of pathogenic MMR variants with previous cancer: a report from the prospective Lynch syndrome database. Gut. 2017;66:1657-64.

59. Tang F, Zheng P. Tumor cells versus host immune cells: whose PD-L1 contributes to PD-1/PD-L1 blockade mediated cancer immunotherapy? Cell Biosci. 2018;8:34.

60. Zhang X, Cheng C, Hou J, Qi X, Wang X, Han P, et al. Distinct contribution of PD-L1 suppression by spatial expression of PD-L1 on tumor and non-tumor cells. Cell Mol Immunol 2018;1-9.

61. Mlecnik B, Bindea G, Angell HK, Maby P, Angelova M, Tougeron D, et al. Integrative Analyses of Colorectal Cancer Show Immunoscore Is a Stronger Predictor of Patient Survival Than Microsatellite Instability. Immunity. 2016;44:698-711.

62. Sorscher S, Resnick J, Goodman M First Case Report of a Dramatic Radiographic Response to a Checkpoint Inhibitor in a Patient With Proficient Mismatch Repair Gene Expressing Metastatic Colorectal Cancer. JCO Precis Oncol 2017;1:1-4. 